Springer Link

\title{
Monetary Intelligence and Behavioral Economics: The Enron Effect-Love of Money, Corporate Ethical Values, Corruption Perceptions Index (CPI), and Dishonesty Across 31 Geopolitical Entities
}

Journal of Business Ethics

April 2018, Volume 148, Issue 4, pp 919-937 | Cite as

- Thomas Li-Ping Tang (1) Email author (Thomas.Tang@mtsu.edu)

- Toto Sutarso (2)

- Mahfooz A. Ansari (3)

- Vivien K. G. Lim (4)

- Thompson S. H. Teo (4)

- Fernando Arias-Galicia (5)

- Ilya E. Garber (6)

- Randy Ki-Kwan Chiu (7)

- Brigitte Charles-Pauvers (8)

- Roberto Luna-Arocas (9)

- Peter Vlerick (10)

- Adebowale Akande (11)

- Michael W. Allen (12)

- Abdulgawi Salim Al-Zubaidi (13)

- Mark G. Borg (14)

- Bor-Shiuan Cheng (15)

- Rosario Correia (16)

- Linzhi Du (17)

- Consuelo Garcia de la Torre (18)

- Abdul Hamid Safwat Ibrahim (19)

- Chin-Kang Jen (20)

- Ali Mahdi Kazem (13)

- Kilsun Kim (21)

- Jian Liang (22)

- Eva Malovics (23)

- Alice S. Moreira (24)

- Richard T. Mpoyi (2)

- Anthony Ugochukwu Obiajulu Nnedum (25)

- Johnsto E. Osagie (26)

- AAhad M. Osman-Gani (27)

- Mehmet Ferhat Özbek (28)

- Francisco José Costa Pereira (29)

- Ruja Pholsward (30)

- Horia D. Pitariu (31) 
- Marko Polic (32)

- Elisaveta Gjorgji Sardžoska (33)

- Petar Skobic (34)

- Allen F. Stembridge (35)

- Theresa Li-Na Tang (36)

- Caroline Urbain (8)

- Martina Trontelj (32)

- Luigina Canova (37)

- Anna Maria Manganelli (37)

- Jingqiu Chen (22)

- Ningyu Tang (22)

- Bolanle E. Adetoun (38)

- Modupe F. Adewuyi (39)

1. Department of Management, Jennings A. Jones College of Business, Middle Tennessee State University, Murfreesboro, USA

2. Middle Tennessee State University, Murfreesboro, USA

3. University of Lethbridge, Lethbridge, Canada

4. National University of Singapore, Singapore, Singapore

5. Universidad Autónoma del Estado de Morelos, Mexico, Mexico

6. Saratov State University, Saratov, Russia

7. Hong Kong Baptist University, Hong Kong, Hong Kong

8. University of Nantes, Nantes, France

9. University of Valencia, Valencia, Spain

10. Ghent University, Ghent, Belgium

11. Independent Research Collaboration, Pretoria, South Africa

12. İpek University, Oran, Turkey

13. Sultan Qaboos University, Muscat, Oman

14. University of Malta, Msida, Malta

15. National Taiwan University, Taipei, Taiwan

16. Polytechnic Institute of Lisbon - Portugal, Lisbon, Portugal

17. Lanzhou University, Lanzhou, China

18. Technological Institute of Monterrey, Monterrey, Mexico

19. Suez Canal University, Ismaileya, Egypt

20. National Sun-Yat-Sen University, Kaohsiung, Taiwan

21. Sogang University, Seoul, South Korea

22. Shanghai Jiao Tong University, Shanghai, China

23. University of Szeged, Szeged, Hungary

24. Federal University of Pará, Castanhal, Brazil

25. Nnamdi Azikiwe University, Awka, Nigeria

26. FAMU, Tallahassee, USA

27. International Islamic University of Malaysia, Kuala Lumpur, Malaysia

28. Gümüşhane University, Gümüşhane, Turkey

29. Lusófona University, Lisbon, Portugal

30. Rangsit University, Pathum Thani, Thailand

31. Babes-Bolyai University, Cluj-Napoca, Romania

32. University of Ljubljana, Ljubljana, Slovenia

33. University St. Cyril and Methodius, Skopje, Macedonia

34. ALDI, Inc., Murfreesboro, USA

35. Andrews Universitv. Berrien Springs. USA 
ט

36. Tang Global Consulting Group, Franklin, USA

37. University of Padua, Padua, Italy

38. Economic Commission of West Africa, Abuja, Nigeria

39. Mercer University, Atlanta, USA

Article

First Online: 27 January 2016

Received: 26 October 2015

Accepted: 02 November 2015

- 840 Downloads

- 8 Citations

\section{Abstract}

Monetary intelligence theory asserts that individuals apply their money attitude to frame critical concerns in the context and strategically select certain options to achieve financial goals and ultimate happiness. This study explores the dark side of monetary Intelligence and behavioral economics-dishonesty (corruption). Dishonesty, a risky prospect, involves cost-benefit analysis of self-interest. We frame good or bad barrels in the environmental context as a proxy of high or low probability of getting caught for dishonesty, respectively. We theorize: The magnitude and intensity of the relationship between love of money and dishonest prospect (dishonesty) may reveal how individuals frame dishonesty in the context of two levels of subjective norm-perceived corporate ethical values at the micro-level (CEV, Level 1) and Corruption Perceptions Index at the macro-level (CPI, Level 2), collected from multiple sources. Based on 6382 managers in 31 geopolitical entities across six continents, our cross-level three-way interaction effect illustrates: As expected, managers in good barrels (high CEV/high CPI), mixed barrels (low CEV/high CPI or high CEV/low CPI), and bad barrels (low CEV/low CPI) display low, medium, and high magnitude of dishonesty, respectively. With high CEV, the intensity is the same across cultures. With low $\mathrm{CEV}$, the intensity of dishonesty is the highest in high CPI entities (risk seeking of high probability)-the Enron Effect, but the lowest in low CPI entities (risk aversion of low probability). CPI has a strong impact on the magnitude of dishonesty, whereas CEV has a strong impact on the intensity of dishonesty. We demonstrate dishonesty in light of monetary values and two frames of social norm, revealing critical implications to the field of behavioral economics and business ethics.

\section{Keywords}

Theory of planned behavior Prospect theory Love of money Behavioral intention/Behavioral ethics Good/bad apples Barrels Risk aversion Risk seeking Cross-cultural Multilevel CPI GDP FDI Global economic pyramid Corruption Human resource management 
Portions of this paper were presented at the Academy of Management Annual Meeting, San Antonio, Texas, August 12-16, 2011.

This is a preview of subscription content, $\underline{\log }$ in to check access.

\section{Notes}

\section{Acknowledgments}

The senior author would like to thank Faculty Research and Creative Activity Committee of Middle Tennessee State University and all co-authors' respective institutions for financial support, late Fr. Wiatt Funk, late Prof. Kuan Ying Tang, and Fang Chen Tang for their inspiration, and pay special tribute to Prof. Horia D. Pitariu who passed away on March 25, 2010.

\section{References}

Adams, J. S. (1963). Toward an understanding of inequity. Journal of Abnormal Social Psychology, 67, 422-436.

CrossRef (https://doi.org/10.1037/hoo40968)

Google Scholar (http://scholar.google.com/scholar_lookup?

title=Toward\%20an\%20understanding\%20of\%20inequity\&author=JS.\%20Adams\&jour nal=Journal\%20of\%20Abnormal\%20Social\%20Psychology\&volume=67\&pages $=422-$

436\&publication_year=1963)

Aiken, L. S., \& West, S. G. (1991). Multiple regression: Testing and interpreting interactions. Newbury Park, CA: Sage.

Google Scholar (http://scholar.google.com/scholar_lookup?

title=Multiple\%2oregression\%3A\%20Testing\%20and\%2ointerpreting\%2ointeractions\& author=LS.\%20Aiken\&author=SG.\%20West\&publication_year=1991)

Ajzen, I. (1991). The theory of planned behavior. Organizational Behavior and Human Decision Processes, 5o, 179-211.

CrossRef (https://doi.org/10.1016/0749-5978(91)90020-T)

Google Scholar (http://scholar.google.com/scholar_lookup?

title=The\%20theory\%20of\%2oplanned\%2obehavior\&author=I.\%20Ajzen\&journal=Orga nizational\%20Behavior\%20and\%20Human\%20Decision\%20Processes\&volume=50\&pa ges $=179-211 \&$ publication_year $=1991$ )

Anand, V., Ashforth, B. E., \& Joshi, M. (2004). Business as usual: The acceptance and perpetuation of corruption in organizations. Academy of Management Executive, 18,

$39-53$.

CrossRef (https://doi.org/10.5465/AME.2004.13837437)

Google Scholar (http://scholar.google.com/scholar_lookup?

title=Business\%20as\%20usual\%3A\%20The\%20acceptance\%20and\%2operpetuation\%2 oof\%20corruption\%20in\%20organizations\&author=V.\%20Anand\&author=BE.\%20Ashf orth\&author=M.\%2OJoshi\&journal=Academy\%20of\%20Management\%20Executive\&vol ume $=18$ \&pages $=39-53 \&$ publication_year $=2004$ ) 
Aquino, K., Freeman, D., Reed, A., Lim, V. K. G., \& Felps, W. (2009). Testing a socialcognitive model of moral behavior: The interactive influence of situations and moral identity centrality. Journal of Personality and Social Psychology, 97, 123-141.

CrossRef (https://doi.org/10.1037/ao015406)

Google Scholar (http://scholar.google.com/scholar_lookup?

title=Testing\%20a\%20social-

cognitive\%20model\%20of\%20moral\%2obehavior\%3A\%20The\%20interactive\%2oinflue nce\%20of\%20situations\%20and\%20moral\%2oidentity\%20centrality\&author=K.\%2OAq uino\&author $=$ D.\%2OFreeman\&author $=$ A.\%20Reed\&author $=$ VKG.\%2OLim\&author $=W$. \%2oFelps\&journal=Journal\%20of\%2oPersonality\%20and\%20Social\%2oPsychology\&vo lume $=97 \&$ pages $=123-141 \&$ publication_year $=2009)$

Ariely, D. (2008a). How honest people cheat. Harvard Business Review, 86(2), 24.

Google Scholar (http://scholar.google.com/scholar_lookup?

title=How\%2ohonest\%2opeople\%20cheat\&author=D.\%20Ariely\&journal=Harvard\%20 Business\%20Review\&volume $=86 \&$ issue $=2 \&$ pages $=24 \&$ publication_year=2008)

Ariely, D. (2008b). Predictably irrational: The hidden forces that shape our decisions. New York: Harper/Perennial.

Google Scholar (http://scholar.google.com/scholar_lookup?

title=Predictably\%20irrational\%3A\%20The\%2ohidden\%20forces\%2othat\%20shape\%20 our\%2odecisions\&author=D.\%20Ariely\&publication_year=2008)

Ariely, D. (2010). You are what you measure. Harvard Business Review, 88(6), 38.

Google Scholar (http://scholar.google.com/scholar_lookup?

title=You\%20are\%20what\%20you\%20measure\&author=D.\%20Ariely\&journal=Harvard $\%$ 2oBusiness $\% 20$ Review\&volume $=88 \&$ issue $=6 \&$ pages $=38 \&$ publication_year $=2010$ )

Ariyabuddhiphongs, V., \& Hongladarom, C. (2011). Violation of Buddhist five percepts, money consciousness, and the tendency to pay bribes among organizational employees in Bangkok, Thailand. Archive for the Psychology of Religion, 33, 325-344.

CrossRef (https://doi.org/10.1163/157361211X594168)

Google Scholar (http://scholar.google.com/scholar_lookup?

title=Violation\%20of\%20Buddhist\%20five\%2opercepts\%2C\%20money\%20consciousne ss\%2C\%20and\%2othe\%2otendency\%20to\%2opay\%2obribes\%20among\%2oorganizatio nal\%20employees\%2oin\%2oBangkok\%2C\%20Thailand\&author=V.\%2OAriyabuddhipho ngs\&author=C.\%20Hongladarom\&journal=Archive\%2ofor\%2othe\%20Psychology\%20of \%20Religion\&volume=33\&pages $=325-344 \&$ publication_year=2011)

Armitage, C. J., \& Conner, M. (2001). Efficacy of the theory of planned behaviour: A meta-analytic review. British Journal of Social Psychology, 4O, 471-499.

CrossRef (https://doi.org/10.1348/014466601164939)

Google Scholar (http://scholar.google.com/scholar_lookup?

title=Efficacy\%20of\%20the\%2otheory\%20of\%2oplanned\%2obehaviour\%3A\%20A\%20 meta-

analytic\%20review\&author=CJ.\%20Armitage\&author=M.\%20Conner\&journal=British\% 20Journal\%20of\%20Social\%20Psychology\&volume $=40 \&$ pages $=471-$

499\&publication_year=2001)

Ashforth, B. E., Gioia, D. A., Robinson, S. L., \& Treviño, L. K. (2008). Introduction to special topic forum: Re-viewing organizational corruption. Academy of Management Review, 33, 670-684.

CrossRef (https://doi.org/10.5465/AMR.2008.32465714) 
Google Scholar (http://scholar.google.com/scholar_lookup?

title=Introduction\%20to\%20special\%20topic\%2oforum\%3A\%2oRe-

viewing\%20organizational\%20corruption\&author=BE.\%20Ashforth\&author=DA.\%20Gi oia\&author=SL.\%20Robinson\&author=LK.\%20Trevi\%C3\%B1o\&journal=Academy\%200 f\%20Management\%20Review\&volume=33\&pages=670-684\&publication_year=2008)

Badaracco, J. L. (2006). Leadership in literature: A conversation with business ethicist Joseph L. Badaracco, Jr. Harvard Business Review, 84(3), 47-55.

Google Scholar (http://scholar.google.com/scholar_lookup?

title=Leadership\%20in\%2oliterature\%3A\%20A\%20conversation\%20with\%2obusiness\% 20ethicist\%2OJoseph\%20L.\%20Badaracco\%2C\%2OJr\&author=JL.\%2oBadaracco\&journ al=Harvard\%20Business\%20Review\&volume $=84$ \&issue $=3$ \&pages $=47^{-}$

55\&publication_year=2006)

Baker, T. L., Hunt, T. G., \& Andrews, M. C. (2006). Promoting ethical behavior and organizational citizenship behaviors: The influence of corporate ethical values. Journal of Business Research, 59(7), 849-857.

CrossRef (https://doi.org/10.1016/j.jbusres.2006.02.004)

Google Scholar (http://scholar.google.com/scholar_lookup?

title=Promoting\%20ethical\%2obehavior\%20and\%2oorganizational\%2ocitizenship\%2ob ehaviors\%3A\%20The\%20influence\%20of\%20corporate\%20ethical\%20values\&author=T L.\%20Baker\&author=TG.\%20Hunt\&author=MC.\%20Andrews\&journal=Journal\%20of\% 20Business $\% 20$ Research \&volume $=59$ \&issue $=7$ \&pages $=849$ -

857\&publication_year=2006)

Bamberger, P. (2008). Beyond contextualization: Using context theories to narrow the micro-macro gap in management research. Academy of Management Journal, 51, 839846.

CrossRef (https://doi.org/10.5465/AMJ.2008.34789630)

Google Scholar (http://scholar.google.com/scholar_lookup?

title=Beyond\%2ocontextualization\%3A\%20Using\%2ocontext\%2otheories\%2oto\%2onar row\%20the\%20micro-

macro\%20gap\%20in\%2omanagement\%2oresearch\&author=P.\%20Bamberger\&journal= Academy\%20of\%20Management\%20Journal\&volume=51\&pages=839-

846\&publication_year=2008)

Bandura, A. (1986). Social foundations of thought and action: A social cognitive theory. Englewood Cliffs, NJ: Prentice Hall.

Google Scholar (http://scholar.google.com/scholar_lookup?

title=Social\%2 ofoundations\%20of\%2othought\%20and\%20action\%3A\%20A\%20social\% 20cognitive\%2otheory\&author=A.\%2oBandura\&publication_year=1986)

Bandura, A., Barbaranelli, C., Caprara, G. V., \& Pastorelli, C. (1996). Mechanisms of moral disengagement in the exercise of moral agency. Journal of Personality and Social Psychology, 71, 364-373.

CrossRef (https://doi.org/10.1037/0022-3514.71.2.364)

Google Scholar (http://scholar.google.com/scholar_lookup?

title=Mechanisms\%20of\%2omoral\%2odisengagement\%20in\%2othe\%20exercise\%2oof

\%20moral\%20agency\&author=A.\%20Bandura\&author $=$ C.\%20Barbaranelli\&author=GV . \%20Caprara\&author=C.\%20Pastorelli\&journal=Journal\%20of\%2oPersonality\%20and\% 20Social\%20Psychology\&volume=71\&pages=364-373\&publication_year=1996)

Bartunek, J. M., Rynes, S. L., \& Ireland, R. D. (2006). What makes management research interesting, and why does it matter? Academy of Management Journal, 49, 9-15. 
CrossRef (https://doi.org/10.5465/AMJ.2006.20785494)

Google Scholar (http://scholar.google.com/scholar_lookup?

title=What\%20makes\%20management\%20research\%2ointeresting\%2C\%20and\%20why \%20does\%20it\%2omatter\%3F\&author=JM.\%2oBartunek\&author=SL.\%20Rynes\&autho $\mathrm{r}=\mathrm{RD} . \% 20$ Ireland\&journal=Academy\%20of\%20Management\%20Journal\&volume=49\& pages $=9-15 \&$ publication_year $=2006$ )

Bateman, T. S., \& Snell, S. A. (2011). Management: Leading \& collaborating in the competitive world (9th ed.). New York: McGraw-Hill/Irwin.

Google Scholar (http://scholar.google.com/scholar_lookup?

title=Management\%3A\%20Leading\%20\%26\%20collaborating\%2oin\%20the\%2ocompeti tive\%20world\&author=TS.\%20Bateman\&author=SA.\%20Snell\&publication_year=2011)

Baumeister, R. F. (2002). Yielding to temptation: Self-control failure, impulsive purchasing, and consumer behavior. Journal of Consumer Research, 28, 670-676. CrossRef (https://doi.org/10.1086/338209)

Google Scholar (http://scholar.google.com/scholar_lookup? title=Yielding\%20to\%20temptation\%3A\%20Selfcontrol\%2ofailure\%2C\%2oimpulsive\%2Opurchasing\%2C\%20and\%2oconsumer\%2obeh avior\&author=RF.\%20Baumeister\&journal=Journal\%20of\%20Consumer\%20Research\& volume $=28 \&$ pages $=670-676 \&$ publication _year $=2002$ )

Belk, R. W. (1985). Materialism: Trait aspects of living in the material world. Journal of Consumer Research, 12, 265-280.

CrossRef (https://doi.org/10.1086/208515)

Google Scholar (http://scholar.google.com/scholar_lookup?

title=Materialism\%3A\%20Trait\%20aspects\%20of\%2oliving\%2oin\%20the\%2omaterial\% 20world\&author=RW.\%2oBelk\&journal=Journal\%20of\%20Consumer\%2oResearch\&vol ume $=12$ \&pages $=265$-280\&publication_year $=1985$ )

Bennett, R. J., \& Robinson, S. L. (2000). Development of a measure of workplace deviance. Journal of Applied Psychology, 85(3), 349-360.

CrossRef (https://doi.org/10.1037/0021-9010.85.3.349)

Google Scholar (http://scholar.google.com/scholar_lookup? title=Development\%20of\%20a\%20measure\%200f\%20workplace\%20deviance\&author= RJ.\%2oBennett\&author=SL.\%20Robinson\&journal=Journal\%20of\%20Applied\%20Psyc hology\&volume $=85 \&$ \&issue $=3$ \&pages $=349-360 \&$ publication_year $=2000$ )

Brislin, R. W. (1980). Translation and content analysis of oral and written materials. In H. C. Triandis \& J. W. Berry (Eds.), Handbook of cross-cultural psychology (Vol. 2: Methodology, pp. 349-444). Boston, MA: Allyn and Bacon.

Google Scholar (http://scholar.google.com/scholar_lookup? title=Translation\%20and\%20content\%20analysis\%20of\%20oral\%20and\%20written\%2 omaterials\&author=RW.\%20Brislin\&publication_year=1980)

Bruner, J. S., \& Goodman, C. C. (1947). Value and needs as organizing factors in perception. Journal of Abnormal and Social Psychology, 42, 33-44.

CrossRef (https://doi.org/10.1037/hoo58484)

Google Scholar (http://scholar.google.com/scholar_lookup? title=Value\%20and\%2oneeds\%20as\%20organizing\%2ofactors\%20in\%2operception\&au thor=JS.\%20Bruner\&author=CC.\%20Goodman\&journal=Journal\%20of\%20Abnormal\% 20and\%20Social\%2oPsychology\&volume $=42 \&$ pages $=33-44 \&$ publication_year $=1947$ ) 
Campbell, J. L. (2007). Why would corporations behave in socially responsible ways? An institutional theory of corporate social responsibility. Academy of Management Review, 32, 946-967.

CrossRef (https://doi.org/10.5465/AMR.2007.25275684)

Google Scholar (http://scholar.google.com/scholar_lookup?

title=Why\%20would\%2ocorporations\%2obehave\%2oin\%20socially\%2oresponsible\%20 ways\%3F\%20An\%20institutional\%20theory\%20of\%20corporate\%20social\%2oresponsi bility\&author=JL.\%20Campbell\&journal=Academy\%20of\%20Management\%20Review\& volume $=32 \&$ pages $=946-967 \&$ publication_year $=2007$ )

Chen, J. Q., Tang, T. L. P., \& Tang, N. Y. (2014). Temptation, monetary intelligence (love of money), and environmental context on unethical intentions and cheating. Journal of Business Ethics, 123(2), 197-219.

CrossRef (https://doi.org/10.1007/s10551-013-1783-2)

Google Scholar (http://scholar.google.com/scholar_lookup?

title=Temptation\%2C\%2omonetary\%20intelligence\%20\%28love\%20of\%2omoney\%29\% 2C\%20and\%20environmental\%20context\%20on\%20unethical\%20intentions\%20and\%2 ocheating\&author=JQ.\%20Chen\&author=TLP.\%20Tang\&author=NY.\%20Tang\&journal =Journal\%20of\%20Business\%20Ethics\&volume=123\&issue=2\&pages=197-

219\&publication_year=2014)

Cheung, G. W., \& Rensvold, R. B. (2002). Evaluating goodness-of-fit indexes for testing measurement invariance. Structural Equation Modeling, 9, 233-255.

CrossRef (https://doi.org/10.1207/S15328007SEM0902_5)

Google Scholar (http://scholar.google.com/scholar_lookup?

title=Evaluating\%20goodness-of-

fit\%20indexes\%20for\%20testing\%20measurement\%20invariance\&author=GW.\%20Che ung\&author=RB.\%20Rensvold\&journal=Structural\%20Equation\%20Modeling\&volume $=9 \&$ pages $=233-255 \&$ publication_year $=2002$ )

Christie, R., \& Geis, F. L. (1970). Studies in Machiavellianism. New York: Academic Press.

Google Scholar (http://scholar.google.com/scholar_lookup?

title=Studies\%20in\%20Machiavellianism\&author=R.\%20Christie\&author=FL.\%20Geis \&publication_year=1970)

Cohen-Charash, Y., \& Spector, P. E. (2001). The role of justice in organizations: A metaanalysis. Organizational Behavior and Human Decision Processes, 86, 278-321.

CrossRef (https://doi.org/10.1006/obhd.2001.2958)

Google Scholar (http://scholar.google.com/scholar_lookup?

title=The\%20role\%20of\%2ojustice\%20in\%20organizations\%3A\%20A\%2ometaanalysis\&author $=$ Y.\%20Cohen-

Charash\&author=PE.\%2oSpector\&journal=Organizational\%2oBehavior\%20and\%2oHu man\%20Decision\%20Processes\&volume=86\&pages=278-321\&publication_year=2001)

Cohn, A., Fehr, E., \& Marechal, M. A. (2014). Business culture and dishonesty in the banking industry. Nature, 516, 86-89.

Google Scholar (http://scholar.google.com/scholar_lookup?

title=Business\%20culture\%20and\%20dishonesty\%20in\%20the\%2obanking\%2oindustry \&author=A.\%20Cohn\&author=E.\%20Fehr\&author=MA.\%20Marechal\&journal=Nature $\&$ volume $=516 \&$ pages $=86-89 \&$ publication_year $=2014$ )

Colquitt, J. A., LePine, J. A., \& Wesson, M. J. (2011). Organizational behavior: Improving performance and commitment in the workplace. New York: McGraw- 
Hill/Irwin.

Google Scholar (http://scholar.google.com/scholar_lookup?

title=Organizational\%2obehavior\%3A\%20Improving\%2operformance\%20and\%20com mitment\%20in\%20the\%20workplace\&author=JA.\%20Colquitt\&author=JA.\%20LePine \&author=MJ.\%20Wesson\&publication_year=2011)

Colquitt, J. A., \& Zapata-Phelan, C. P. (2007). Trends in theory building and theory testing: A five-decade study of the Academy of Management Journal. Academy of Management Journal, 5o, 1281-1303.

CrossRef (https://doi.org/10.5465/AMJ.2007.28165855)

Google Scholar (http://scholar.google.com/scholar_lookup?

title=Trends\%20in\%20theory\%2obuilding\%20and\%2otheory\%2otesting\%3A\%20A\%2of ive-

decade\%20study\%20of\%20the\%20Academy\%200f\%20Management\%20Journal\&autho $\mathrm{r}=\mathrm{JA} . \% 20$ Colquitt\&author=CP.\%20Zapata-

Phelan\&journal=Academy\%20of\%20Management\%20Journal\&volume=50\&pages $=1281$ -1303\&publication_year=2007)

Cordano, M., \& Frieze, I. H. (2000). Pollution reduction preferences of US environmental managers: Applying Ajzen's theory of planned behavior. Academy of Management Journal, 43, 627-641.

CrossRef (https://doi.org/10.2307/1556358)

Google Scholar (http://scholar.google.com/scholar_lookup?

title=Pollution\%2oreduction\%2opreferences\%20of\%20US\%2oenvironmental\%2omana gers\%3A\%20Applying\%20Ajzen\%E2\%80\%99s\%20theory\%20of\%2oplanned\%2obehavi or\&author=M.\%20Cordano\&author=IH.\%20Frieze\&journal=Academy\%20of\%20Manag ement\%20Journal\&volume=43\&pages=627-641\&publication_year=2000)

Cozzolino, P. J., Sheldon, K. M., Schachtman, T. R., \& Meyers, L. S. (2009). Limited time perspective, values, and greed: Imagining a limited future reduces avarice in extrinsic people. Journal of Research in Personality, 43, 399-408.

CrossRef (https://doi.org/10.1016/j.jrp.2009.01.008)

Google Scholar (http://scholar.google.com/scholar_lookup?

title=Limited\%2otime\%2operspective\%2C\%20values\%2C\%20and\%2ogreed\%3A\%20Im agining\%20a\%2olimited\%2ofuture\%2oreduces\%20avarice\%2oin\%20extrinsic\%2opeopl e\&author=PJ.\%20Cozzolino\&author=KM.\%20Sheldon\&author=TR.\%20Schachtman\&a uthor=LS.\%20Meyers\&journal=Journal\%20of\%20Research\%20in\%20Personality\&volu $\mathrm{me}=43$ \&pages $=399-408 \&$ publication_year $=2009$ )

Davis, J. H., Schoorman, F. D., \& Donaldson, L. (1997). Toward a stewardship theory of management. Academy of Management Review, 22, 20-47.

Google Scholar (http://scholar.google.com/scholar_lookup?

title=Toward\%20a\%20stewardship\%20theory\%20of\%2omanagement\&author=JH.\%20 Davis\&author=FD.\%20Schoorman\&author $=$ L.\%20Donaldson\&journal=Academy\%20of \%20Management\%20Review\&volume=22\&pages=20-47\&publication_year=1997)

Dawson, J. F., \& Richter, A. W. (2006). Probing three-way interactions in moderated multiple regression: Development and application of a slope difference test. Journal of Applied Psychology, 91(4), 917-926.

CrossRef (https://doi.org/10.1037/0021-9010.91.4.917)

Google Scholar (http://scholar.google.com/scholar_lookup?title=Probing\%20threeway\%20interactions\%20in\%20moderated\%20multiple\%2oregression\%3A\%20Develop ment\%20and\%20application\%20of\%20a\%20slope\%20difference\%2otest\&author=JF.\% 
20Dawson\&author=AW.\%20Richter\&journal=Journal\%20of\%20Applied\%2oPsycholog y\&volume $=91 \&$ issue $=4 \&$ pages $=917-926 \&$ publication_year $=2006$ )

De Jonge, J., \& Peeters, M. C. W. (2009). Convergence of self-reports and coworker reports of counterproductive work behavior: A cross-sectional multi-source survey among health care workers. International Journal of Nursing Studies, 46, 699-707. CrossRef (https://doi.org/10.1016/j.ijnurstu.2008.12.010)

Google Scholar (http://scholar.google.com/scholar_lookup? title $=$ Convergence $\% 200 f \% 20$ selfreports\%20and\%20coworker\%2oreports\%20of\%2ocounterproductive\%20work\%2obeh avior\%3A\%20A\%20cross-sectional\%2omulti-

source\%20survey\%20among\%2ohealth\%20care\%20workers\&author=J.\%2OJonge\&auth or=MCW.\%2oPeeters\&journal=International\%20Journal\%20of\%20Nursing\%20Studies \&volume $=46 \&$ pages $=699-707 \&$ publication_year $=2009)$

Dineen, B. R., Lewicki, R. J., \& Tomlinson, E. C. (2006). Supervisory guidance and behavioral integrity: Relationships with employee citizenship and deviant behavior. Journal of Applied Psychology, 91, 622-635. CrossRef (https://doi.org/10.1037/0021-9010.91.3.622) Google Scholar (http://scholar.google.com/scholar_lookup? title=Supervisory\%2oguidance\%20and\%2obehavioral\%2ointegrity\%3A\%2oRelationship s\%20with\%20employee\%2ocitizenship\%20and\%2odeviant\%2obehavior\&author=BR.\% 2oDineen\&author=RJ.\%20Lewicki\&author=EC.\%20Tomlinson\&journal=Journal\%20of \%20Applied\%20Psychology\&volume=91\&pages=622-635\&publication_year=2006)

Drori, G. S., Jang, Y. S., \& Meyer, J. W. (2006). Sources of rationalized governance: Cross-national longitudinal analyses 1985-2002. Administrative Science Quarterly, 51, 205-229.

CrossRef (https://doi.org/10.2189/asqu.51.2.205)

Google Scholar (http://scholar.google.com/scholar_lookup? title=Sources\%20of\%2orationalized\%2ogovernance\%3A\%20Cross-

national\%2olongitudinal\%20analyses\%201985\%E2\%80\%932002\&author=GS.\%20Dror i\&author=YS.\%20Jang\&author=JW.\%2oMeyer\&journal=Administrative\%2oScience\%2 oQuarterly\&volume=51\&pages $=205-229 \&$ publication_year $=2006$ )

Easterlin, R. A. (2001). Income and happiness: Towards a unified theory. The Economic Journal, 111, 465-484.

CrossRef (https://doi.org/10.1111/1468-0297.00646)

Google Scholar (http://scholar.google.com/scholar_lookup?

title=Income\%20and\%2ohappiness\%3A\%20Towards\%20a\%20unified\%2otheory\&auth or $=$ RA.\%20Easterlin\&journal $=$ The $\% 20$ Economic\%20Journal\&volume $=111 \&$ pages $=465$ 484\&publication_year=2001)

Erdener, C., \& Garkavenko, V. (2012). Money attitudes in Kazakhstan. Journal of International Business and Economics, 12(3), 87-94.

Google Scholar (http://scholar.google.com/scholar_lookup?

title=Money\%20attitudes\%20in\%20Kazakhstan\&author=C.\%2oErdener\&author=V.\%20 Garkavenko\&journal=Journal\%20of\%2OInternational\%2oBusiness\%20and\%2oEconom ics\&volume $=12 \&$ issue $=3 \&$ pages $=87-94 \&$ publication_year $=2012)$

Exadaktylos, F., Espin, A. M., \& Branas-Garza, P. (2013). Experimental subjects are not different. Scientific Reports, 3, 1213. doi: 10.1038/srepo1213

(https://doi.org/10.1038/srepo1213).

CrossRef (https://doi.org/10.1038/srepo1213) 
Google Scholar (http://scholar.google.com/scholar_lookup?

title=Experimental\%20subjects\%20are\%2onot\%2odifferent\&author=F.\%2oExadaktylos \&author=AM.\%20Espin\&author=P.\%20Branas-

Garza\&journal=Scientific\%20Reports\&volume=3\&pages=1213\&publication_year=2013 \&doi $=10.1038 \% 2$ Fsrep01213)

Feiner, M. (2004). The Feiner points of leadership. New York: Warner Business Books.

Google Scholar (http://scholar.google.com/scholar_lookup?

title=The\%20Feiner\%20points\%20of\%2oleadership\&author=M.\%20Feiner\&publication _year=2004)

Fisman, R., \& Miguel, E. (2007). Corruption, norms, and legal enforcement: Evidence from diplomatic parking tickets. Journal of Political Economy, 115(6), 1020-1048.

CrossRef (https://doi.org/10.1086/527495)

Google Scholar (http://scholar.google.com/scholar_lookup?

title=Corruption\%2C\%20norms\%2C\%20and\%2olegal\%2oenforcement\%3A\%2oEvidenc e\%2ofrom\%20diplomatic\%2oparking\%20tickets\&author=R.\%2oFisman\&author=E.\%2 oMiguel\&journal=Journal\%20of\%20Political\%20Economy\&volume=115\&issue=6\&page S=1020-1048\&publication_year=2007)

Fox, S., Spector, P. E., Goh, A., \& Bruursema, K. (2007). Does your coworker know what you're doing? Convergence of self- and peer-reports of counterproductive work behavior. International Journal of Stress Management, 14, 41-60.

CrossRef (https://doi.org/10.1037/1072-5245.14.1.41)

Google Scholar (http://scholar.google.com/scholar_lookup? title=Does\%20your\%20coworker\%20know\%20what\%20you\%E2\%80\%99re\%20doing\% 3F\%20Convergence\%20of\%20self-\%20and\%2opeer-

reports\%20of\%20counterproductive\%20work\%20behavior\&author=S.\%20Fox\&author= PE.\%20Spector\&author=A.\%20Goh\&author=K.\%20Bruursema\&journal=International\% 20Journal\%20of\%20Stress\%20Management\&volume=14\&pages $=41-$

6o\&publication_year=2007)

Frey, B. S., \& Jegen, R. (2001). Motivation crowding theory. Journal of Economic

Surveys, 15, 589-611.

CrossRef (https://doi.org/10.1111/1467-6419.00150)

Google Scholar (http://scholar.google.com/scholar_lookup?

title=Motivation\%20crowding\%20theory\&author=BS.\%20Frey\&author=R.\%20Jegen\&jo urnal=Journal\%20of\%20Economic\%20Surveys\&volume=15\&pages=589-

611\&publication_year=2001)

Furnham, A. (2014). The new psychology of money. London: Routledge.

Google Scholar (http://scholar.google.com/scholar_lookup?

title=The\%20new\%20psychology\%200f\%20money\&author=A.\%20Furnham\&publicatio n_year=2014)

Furnham, A., \& Argyle, M. (1998). The psychology of money. London: Routledge. Google Scholar (http://scholar.google.com/scholar_lookup? title=The\%20psychology\%20of\%20money\&author=A.\%20Furnham\&author=M.\%20Arg yle\&publication_year=1998)

Gachter, S. (2015). Behavioral economics: Visible inequality breeds more inequality.

Nature, 526(7573), 333-334.

CrossRef (https://doi.org/10.1038/526333a) 
Google Scholar (http://scholar.google.com/scholar_lookup?

title=Behavioral\%20economics\%3A\%20Visible\%2oinequality\%2obreeds\%2omore\%2oin equality\&author $=$ S.\%20Gachter\&journal $=$ Nature $\&$ volume $=526 \&$ issue $=7573 \&$ pages $=333$ -334\&publication_year=2015)

Gbadamosi, G., \& Joubert, P. (2005). Money ethic, moral conduct and work related attitudes: Field study from the public sector in Swaziland. Journal of Management Development, 24, 754-763.

CrossRef (https://doi.org/10.1108/02621710510613762)

Google Scholar (http://scholar.google.com/scholar_lookup?

title=Money\%20ethic\%2C\%20moral\%20conduct\%20and\%20work\%2orelated\%20attitu des\%3A\%2oField\%20study\%20from\%20the\%2opublic\%2osector\%20in\%2oSwaziland\& author=G.\%20Gbadamosi\&author=P.\%20Joubert\&journal=Journal\%20of\%20Manage ment\%20Development\&volume=24\&pages $=754-763 \&$ publication_year=2005)

Gentina, E., Tang, T. L. P., \& Gu, Q. X. (2015). Does bad company corrupt good morals? Social bonding and academic cheating among French and Chinese teens. Journal of Business Ethics. doi: 10.1007/s10551-015-2939-z (https://doi.org/10.1007/s10551-0152939-z).

Google Scholar (http://scholar.google.com/scholar_lookup?

title=Does\%2obad\%20company\%20corrupt\%2ogood\%20morals\%3F\%20Social\%2obon ding\%20and\%20academic\%20cheating\%20among\%2oFrench\%20and\%20Chinese\%20t eens\&author=E.\%20Gentina\&author=TLP.\%20Tang\&author=QX.\%20Gu\&journal=Jour nal\%20of\%20Business\%20Ethics\&publication_year=2015\&doi=10.1007\%2Fs10551-0152939-z)

Gilbert, J. A., \& Tang, T. L. P. (1998). An examination of organizational trust antecedents. Public Personnel Management, 27, 321-338. CrossRef (https://doi.org/10.1177/009102609802700303) Google Scholar (http://scholar.google.com/scholar_lookup? title=An\%20examination\%20of\%20organizational\%20trust\%20antecedents\&author=JA \%20Gilbert\&author=TLP.\%20Tang\&journal=Public\%2oPersonnel\%2oManagement\&vo lume $=27 \&$ pages $=321-338 \&$ publication $\_$year $\left.=1998\right)$

Gino, F., \& Pierce, L. (2009a). Dishonesty in the name of equity. Psychological Science, 2O(9), 1153-1160.

CrossRef (https://doi.org/10.1111/j.1467-9280.2009.02421.x)

Google Scholar (http://scholar.google.com/scholar_lookup?

title=Dishonesty\%20in\%20the\%20name\%20of\%20equity\&author=F.\%20Gino\&author= L.\%20Pierce\&journal=Psychological\%20Science\&volume=20\&issue=9\&pages $=1153$ 1160\&publication_year=2009)

Gino, F., \& Pierce, L. (2009b). The abundance effect: Unethical behavior in the presence of wealth. Organizational Behavior and Human Decision Processes, 109, 142-155.

CrossRef (https://doi.org/10.1016/j.obhdp.2009.03.003)

Google Scholar (http://scholar.google.com/scholar_lookup?

title=The\%20abundant\%20effect\%3A\%20Unethical\%2obehavior\%20in\%20the\%2opres ence\%20of\%20wealth\&author=F.\%20Gino\&author=L.\%20Pierce\&journal=Organization al\%20Behavior\%20and\%20Human\%20Decision\%20Processes\&volume=109\&pages=14 2-155\&publication_year=2009)

Gomez-Mejia, L., Wiseman, R. M., \& Dykes, B. J. (2005). Agency problems in diverse contexts: A global perspective. Journal of Management Studies, 42, 1507-1517.

CrossRef (https://doi.org/10.1111/j.1467-6486.2005.00554.x) 
Google Scholar (http://scholar.google.com/scholar_lookup?

title=Agency\%2oproblems\%20in\%20diverse\%2ocontexts\%3A\%2oA\%2oglobal\%2opersp ective\&author $=$ L.\%20Gomez-

Mejia\&author=RM.\%20Wiseman\&author=BJ.\%20Dykes\&journal=Journal\%20of\%20M anagement\%20Studies\&volume $=42 \&$ pages $=1507-1517 \&$ publication_year $=2005$ )

Gorodnichenko, Y., \& Peter, K. S. (2007). Public sector pay and corruption: Measuring bribery from micro data. Journal of Public Economics, 91, 963-991.

CrossRef (https://doi.org/10.1016/j.jpubeco.2006.12.003)

Google Scholar (http://scholar.google.com/scholar_lookup?

title=Public\%20sector\%2opay\%20and\%20corruption\%3A\%2oMeasuring\%2obribery\%2 ofrom\%20micro\%20data\&author=Y.\%20Gorodnichenko\&author=KS.\%2OPeter\&journal =Journal\%200f\%20Public\%20Economics\&volume=91\&pages $=963-$

991\&publication_year=2007)

Grant, A. M. (2008). Does intrinsic motivation fuel the prosocial fire? Motivational synergy in predicting persistence, performance, and productivity. Journal of Applied Psychology, 93, 48.

CrossRef (https://doi.org/10.1037/0021-9010.93.1.48)

Google Scholar (http://scholar.google.com/scholar_lookup?

title=Does\%20intrinsic\%20motivation\%2ofuel\%20the\%2oprosocial\%2ofire\%3F\%20Mo tivational\%20synergy\%20in\%2opredicting\%2opersistence\%2C\%2operformance\%2C\%2 oand\%2oproductivity\&author=AM.\%20Grant\&journal=Journal\%20of\%20Applied\%20P sychology\&volume=93\&pages=48\&publication_year=2008)

Greenberg, J. (1993). Stealing in the name of justice: Informational and interpersonal moderators of theft reactions to underpayment inequity. Organizational Behavior and Human Decision Processes, 54, 81-103.

CrossRef (https://doi.org/10.1006/obhd.1993.1004)

Google Scholar (http://scholar.google.com/scholar_lookup?

title=Stealing\%20in\%20the\%20name\%20of\%20justice\%3A\%20Informational\%20and\% 2ointerpersonal\%20moderators\%20of\%2otheft\%2oreactions\%20to\%2ounderpayment\% 2oinequity\&author=J.\%20Greenberg\&journal=Organizational\%20Behavior\%20and\%20 Human\%20Decision\%20Processes\&volume $=54$ \&pages $=81$ -

103\&publication_year=1993)

Greenberg, J. (2002). Who stole the money, and when? Individual and situational determinants of manager theft. Organizational Behavior and Human Decision

Processes, 89, 985-1003.

CrossRef (https://doi.org/10.1016/So749-5978(02)ooo39-0)

Google Scholar (http://scholar.google.com/scholar_lookup?

title=Who\%20stole\%2othe\%20money\%2C\%20and\%20when\%3F\%2oIndividual\%2oand \%2osituational\%20determinants\%20of\%20manager\%20theft\&author=J.\%20Greenberg \&journal=Organizational\%2oBehavior\%20and\%20Human\%20Decision\%20Processes\&v olume $=89$ \&pages $=985-1003 \&$ publication_year=2002)

Grossman, H. I. (1999). Kleptocracy and revolutions. Oxford Economic Papers, 51, 267283.

CrossRef (https://doi.org/10.1093/oep/51.2.267)

Google Scholar (http://scholar.google.com/scholar_lookup?

title=Kleptocracy\%20and\%2orevolutions\&author=HI.\%20Grossman\&journal=Oxford\%

20Economic\%20Papers\&volume $=51 \&$ pages $=267-283 \&$ publication_year $=1999$ ) 
Herzberg, F. (1987). One more time: How do you motivate employees? Harvard Business Review, 65(5), 109-120.

Google Scholar (http://scholar.google.com/scholar_lookup?

title=One\%20more\%20time\%3A\%20How\%20do\%20you\%2omotivate\%20employees\%3 F\&author $=$ F.\%20Herzberg\&journal $=$ Harvard\%20Business\%20Review\&volume $=65$ \&issu e=5\&pages $=109-120 \&$ publication_year $=1987$ )

Hitt, M. A., Beamish, P. W., Jackson, S. E., \& Mathieu, J. E. (2007). Building theoretical and empirical bridges across levels: Multilevel research in management. Academy of Management Journal, 5o, 1385-1399.

CrossRef (https://doi.org/10.5465/AMJ.2007.28166219)

Google Scholar (http://scholar.google.com/scholar_lookup?

title=Building\%20theoretical\%20and\%2oempirical\%2obridges\%20across\%2olevels\%3A \%2oMultilevel\%2oresearch\%20in\%2omanagement\&author=MA.\%20Hitt\&author=PW.

\%20Beamish\&author=SE.\%20Jackson\&author=JE.\%20Mathieu\&journal=Academy\%20 of\%20Management\%20Journal\&volume $=50$ \&pages $=1385^{-}$

1399\&publication_year=2007)

Hsee, C. K., Yang, Y., Li, N., \& Shen, L. X. (2009). Wealth, warmth, and well-being: Whether happiness is relative or absolute depends on whether it is about money, acquisition, or consumption. Journal of Marketing Research, 46, 396-409.

CrossRef (https://doi.org/10.1509/jmkr.46.3.396)

Google Scholar (http://scholar.google.com/scholar_lookup?

title=Wealth\%2C\%20warmth\%2C\%20and\%20well-

being\%3A\%2oWhether\%2ohappiness\%20is\%2orelative\%20or\%20absolute\%2odepends \%20on\%2Owhether\%20it\%20is\%20about\%2omoney\%2C\%20acquisition\%2C\%20or\%2 oconsumption\&author $=\mathrm{CK} . \% 20$ Hsee\&author $=\mathrm{Y} . \% 20$ Yang\&author $=\mathrm{N} . \% 20$ Li\&author $=\mathrm{L}$ $\mathrm{X} . \% 20$ Shen\&journal=Journal\%20of\%20Marketing\%20Research\&volume=46\&pages $=39$ 6-409\&publication_year=2009)

Hunt, S. D., Wood, V. R., \& Chonko, L. B. (1989). Corporate ethical values and Organizational Commitment in Marketing. Journal of Marketing, 53, 79-90.

CrossRef (https://doi.org/10.2307/1251344)

Google Scholar (http://scholar.google.com/scholar_lookup?

title=Corporate\%20ethical\%20values\%20and\%20Organizational\%20Commitment\%20i n\%20Marketing\&author=SD.\%20Hunt\&author=VR.\%20Wood\&author=LB.\%20Chonko \&journal=Journal\%20of\%20Marketing\&volume $=53$ \&pages $=79-$

90\&publication_year=1989)

Ivancevich, J. M., Konopaske, R., \& Matteson, M. T. (2005). Organizational behavior and management (7th ed.). Boston, MA: McGraw-Hill Irwin.

Google Scholar (http://scholar.google.com/scholar_lookup? title=Organizational\%2obehavior\%20and\%2omanagement\&author=JM.\%20Ivancevich \&author=R.\%20Konopaske\&author=MT.\%20Matteson\&publication_year=2005)

Jenkins, G. D., Mitra, A., Gupta, N., \& Shaw, D. (1998). Are financial incentives related to performance? A meta-analytic review of empirical research. Journal of Applied

Psychology, 83, 777-787.

CrossRef (https://doi.org/10.1037/0021-9010.83.5.777)

Google Scholar (http://scholar.google.com/scholar_lookup?

title=Are\%20financial\%2oincentives\%2orelated\%20to\%2operformance\%3F\%20A\%20m eta-

analytic\%2oreview\%20of\%20empirical\%2oresearch\&author=GD.\%20Jenkins\&author= 
A.\%20Mitra\&author=N.\%20Gupta\&author=D.\%20Shaw\&journal=Journal\%20of\%20Ap plied\%20Psychology\&volume=83\&pages=777-787\&publication_year=1998)

Kahneman, D. (2011). Thinking, fast and slow. New York: Farrar, Strass and Giroux. Google Scholar (http://scholar.google.com/scholar_lookup?

title=Thinking\%2C\%20fast\%20and\%20slow\&author=D.\%20Kahneman\&publication_ye $\operatorname{ar}=2011$ )

Kahneman, D., \& Deaton, A. (2010). High income improves evaluation of life but not emotional well-being. Proceedings of the National Academy of Sciences of the United States of America, 107(38), 16489-16493.

CrossRef (https://doi.org/10.1073/pnas.1011492107)

Google Scholar (http://scholar.google.com/scholar_lookup?

title=High\%20income\%20improves\%20evaluation\%20of\%2olife\%2obut\%20not\%20em otional\%20well-

being\&author=D.\%20Kahneman\&author=A.\%20Deaton\&journal=Proceedings\%20of\%2 othe\%2oNational\%20Academy\%20of\%20Sciences\%20of\%2othe\%2oUnited\%20States\% 20of\%20America\&volume $=107 \&$ issue $=38 \&$ pages $=16489$ -

16493\&publication_year=2010)

Kahneman, D., \& Tversky, A. (1979). Prospect theory: An analysis of decision under risk. Econometrica, 47, 263-291.

CrossRef (https://doi.org/10.2307/1914185)

Google Scholar (http://scholar.google.com/scholar_lookup?

title=Prospect\%20theory\%3A\%20An\%20analysis\%20of\%2odecision\%20under\%2orisk\& author=D.\%20Kahneman\&author=A.\%20Tversky\&journal=Econometrica\&volume $=47 \&$ pages $=263-291 \&$ publication_year=1979)

Kasser, T. (2002). The high price of materialism. Cambridge, MA: MIT Press.

Google Scholar (http://scholar.google.com/scholar_lookup?

title=The\%2ohigh\%2oprice\%20of\%2omaterialism\&author=T.\%2oKasser\&publication_y ear=2002)

Kirkman, B., \& Law, K. (2005). International management research in AMJ: Our past, present, and future. Academy of Management Journal, 48, 377-386.

CrossRef (https://doi.org/10.5465/AMJ.2005.17407902)

Google Scholar (http://scholar.google.com/scholar_lookup?

title=International\%20management\%2oresearch\%20in\%20AMJ\%3A\%20Our\%2opast\% 2C\%2opresent\%2C\%20and\%2ofuture\&author=B.\%20Kirkman\&author=K.\%2OLaw\&jou rnal=Academy\%20of\%20Management\%20Journal\&volume=48\&pages $=377-$

386\&publication_year=2005)

Kish-Gephart, J. J., Harrison, D. A., \& Treviño, L. K. (2010). Bad apples, bad cases, and bad barrels: Meta-analytic evidence about sources of unethical decisions at work.

Journal of Applied Psychology, 95, 1-31.

CrossRef (https://doi.org/10.1037/ao017103)

Google Scholar (http://scholar.google.com/scholar_lookup?

title=Bad\%20apples\%2C\%2obad\%20cases\%2C\%20and\%2obad\%2obarrels\%3A\%2oMet a-

analytic\%20evidence\%20about\%20sources\%20of\%2ounethical\%2odecisions\%20at\%20 work\&author=JJ.\%20Kish-

Gephart\&author=DA.\%20Harrison\&author=LK.\%20Trevi\%C3\%B1o\&journal=Journal\% 200f\%20Applied\%20Psychology\&volume=95\&pages $=1-31 \&$ publication_year=2010) 
Klotz, A. C., \& Bolino, M. C. (2013). Citizenship and counterproductive work behavior: A moral licensing view. Academy of Management Review, 38, 292-306.

CrossRef (https://doi.org/10.5465/amr.2011.0109)

Google Scholar (http://scholar.google.com/scholar_lookup?

title=Citizenship\%20and\%2ocounterproductive\%20work\%2obehavior\%3A\%20A\%20mo ral\%2olicensing\%20view\&author=AC.\%20Klotz\&author=MC.\%2oBolino\&journal=Acad emy\%20of\%20Management\%20Review\&volume=38\&pages=292-

306\&publication_year=2013)

Lange, D. (2008). A multidimensional conceptualization of organizational corruption control. Academy of Management Review, 33, 710-729.

CrossRef (https://doi.org/10.5465/AMR.2008.32465742)

Google Scholar (http://scholar.google.com/scholar_lookup?

title=A\%2omultidimensional\%20conceptualization\%20of\%20organizational\%2ocorrupt ion\%20control\&author=D.\%20Lange\&journal=Academy\%20of\%20Management\%20Re view\&volume $=33 \&$ pages $=710-729 \&$ publication $\_$year $=2008$ )

Lawler, E. E. (1971). Pay and organizational effectiveness: A psychological view. New York: McGraw-Hill.

Google Scholar (http://scholar.google.com/scholar_lookup?

title=Pay\%20and\%20organizational\%2oeffectiveness\%3A\%20A\%20psychological\%20vi ew\&author=EE.\%2oLawler\&publication_year=1971)

Lea, S. E. G., \& Webley, P. (2006). Money as tool, money as drug: The biological psychology of a strong incentive. Behavioral and Brain Sciences, 29, 161-209.

Google Scholar (http://scholar.google.com/scholar_lookup?

title=Money\%20as\%20tool\%2C\%20money\%20as\%2odrug\%3A\%2oThe\%2obiological\%2 opsychology\%20of\%20a\%20strong\%20incentive\&author=SEG.\%20Lea\&author=P.\%20 Webley\&journal=Behavioral\%20and\%20Brain\%20Sciences\&volume=29\&pages $=161$ 209\&publication_year=2006)

Lemrová, S., Reiterová, E., Fatěnová, R., Lemr, K., \& Tang, T. L. P. (2014). Money is power: Monetary intelligence-love of money and temptation of materialism among Czech university students. Journal of Business Ethics, 125(2), 329-348.

CrossRef (https://doi.org/10.1007/s10551-013-1915-8)

Google Scholar (http://scholar.google.com/scholar_lookup?

title=Money\%20is\%2opower\%3A\%20Monetary\%2ointelligence\%E2\%80\%94love\%20of \%20money\%20and\%20temptation\%20of\%2omaterialism\%20among\%20Czech\%20univ ersity\%2ostudents\&author=S.\%20Lemrov\%C3\%A1\&author=E.\%20Reiterov\%C3\%A1\&au thor=R.\%20Fat\%C4\%9Bnov\%C3\%A1\&author=K.\%20Lemr\&author=TLP.\%20Tang\&jour nal $=$ Journal\%20of\%20Business $\% 20$ Ethics\&volume $=125 \&$ issue $=2 \&$ pages $=329-$

348\&publication_year=2014)

Levine, D. P. (2005). The corrupt organization. Human Relations, 58, 723-740.

CrossRef (https://doi.org/10.1177/0018726705057160)

Google Scholar (http://scholar.google.com/scholar_lookup?

title=The\%20corrupt\%20organization\&author=DP.\%20Levine\&journal=Human\%20Rel ations\&volume $=58 \&$ pages $=723-740 \&$ publication $\_$year $=2005$ )

Lim, V. K. G., \& Sng, Q. S. (2006). Does parental job insecurity matter? Money anxiety, money motives, and work motivation. Journal of Applied Psychology, 91, 1078-1087.

CrossRef (https://doi.org/10.1037/0021-9010.91.5.1078)

Google Scholar (http://scholar.google.com/scholar_lookup?

title=Does\%20parental\%20job\%20insecurity\%20matter\%3F\%20Money\%20anxiety\%2C 
\%20money\%2omotives\%2C\%20and\%20work\%20motivation\&author=VKG.\%20Lim\&au thor=QS.\%20Sng\&journal=Journal\%20of\%20Applied\%20Psychology\&volume=91\&page S=1078-1087\&publication_year=2006)

Lim, V. K. G., \& Teo, T. S. H. (1997). Sex, money and financial hardship: An empirical study of attitudes towards money among undergraduates in Singapore. Journal of Economic Psychology, 18, 369-386.

CrossRef (https://doi.org/10.1016/So167-4870(97)0o013-5) Google Scholar (http://scholar.google.com/scholar_lookup? title=Sex\%2C\%2omoney\%20and\%2ofinancial\%2ohardship\%3A\%20An\%2oempirical\%2 ostudy\%20of\%20attitudes\%20towards\%20money\%20among\%2oundergraduates\%2oin \%20Singapore\&author=VKG.\%20Lim\&author=TSH.\%20Teo\&journal=Journal\%20of\%2 oEconomic\%2oPsychology\&volume=18\&pages=369-386\&publication_year=1997)

Litzky, B. E., Eddleston, K. A., \& Kidder, D. L. (2006). The good, the bad, and the misguided: How managers inadvertently encourage deviant behaviors. Academy of Management Perspectives, 20, 91-103.

CrossRef (https://doi.org/10.5465/AMP.2006.19873411)

Google Scholar (http://scholar.google.com/scholar_lookup?

title=The\%20good\%2C\%20the\%2obad\%2C\%20and\%20the\%2omisguided\%3A\%20How \%20managers\%2oinadvertently\%20encourage\%2odeviant\%2obehaviors\&author=BE.\% 20Litzky\&author=KA.\%2oEddleston\&author=DL.\%20Kidder\&journal=Academy\%20of \%20Management\%20Perspectives\&volume=20\&pages $=91-$

103\&publication_year=2006)

Liu, B. C., \& Tang, T. L. P. (2011). Does the love of money moderate the relationship between public service motivation and job satisfaction? The case of Chinese professionals in the public sector. Public Administration Review, 71(5), 718-727.

CrossRef (https://doi.org/10.1111/j.1540-6210.2011.02411.x) Google Scholar (http://scholar.google.com/scholar_lookup? title=Does\%20the\%2olove\%20of\%2omoney\%2omoderate\%2othe\%2orelationship\%2ob etween\%20public\%20service\%2omotivation\%20and\%20job\%20satisfaction\%3F\%20Th e\%20case\%20of\%20Chinese\%2oprofessionals\%20in\%20the\%2opublic\%20sector\&auth or=BC.\%2oLiu\&author=TLP.\%20Tang\&journal=Public\%20Administration\%2oReview\& volume $=71 \&$ issue $=5 \&$ pages $=718-727 \&$ publication_year $=2011$ )

Locke, E. A., Feren, D. B., McCaleb, V. M., Shaw, K. N., \& Denny, A. T. (1980). The relative effectiveness of four methods of motivating manager performance. In K. D. Duncan, M. M. Gruneberg, \& D. Wallis (Eds.), Changes in working life (pp. 363-388). New York: Wiley.

Google Scholar (http://scholar.google.com/scholar_lookup?

title=The\%20relative\%20effectiveness\%20of\%2ofour\%20methods\%20of\%2omotivating \%20manager\%2operformance\&author=EA.\%20Locke\&author=DB.\%20Feren\&author= VM.\%20McCaleb\&author=KN.\%20Shaw\&author=AT.\%2oDenny\&pages=363388\&publication_year=1980)

Malhotra, D., \& Gino, F. (2011). The pursuit of power corrupts: How investing in outside options motivates opportunism in relationship. Administrative Science Quarterly,

56(SI), 559-592.

CrossRef (https://doi.org/10.1177/0001839212441350)

Google Scholar (http://scholar.google.com/scholar_lookup?

title=The\%2opursuit\%20of\%2opower\%20corrupts\%3A\%20How\%2oinvesting\%20in\%2 ooutside\%20options\%2omotivates\%20opportunism\%20in\%2orelationship\&author=D. 
\%20Malhotra\&author=F.\%20Gino\&journal=Administrative\%2oScience\%20Quarterly\&v olume $=56$ \&issue $=$ SI\&pages $=559-592 \&$ publication $\_$year $=2011$ )

Manning, M. (2009). The effects of subjective norms on behaviour in the theory of planned behaviour: A meta-analysis. British Journal of Social Psychology, 48, 649-705. CrossRef (https://doi.org/10.1348/014466608X393136)

Google Scholar (http://scholar.google.com/scholar_lookup? title=The\%20effects\%20of\%20subjective\%20norms\%20on\%2obehaviour\%20in\%20the \%2otheory\%20of\%2oplanned\%2obehaviour\%3A\%20A\%2ometaanalysis\&author=M.\%20Manning\&journal=British\%20Journal\%20of\%20Social\%20Psy chology\&volume=48\&pages $=649-705 \&$ publication_year=2009)

Martin, K. D., \& Cullen, J. B. (2006). Continuities and extensions of ethical climate theory: A meta-analytic review. Journal of Business Ethics, 69, 175-194.

CrossRef (https://doi.org/10.1007/s10551-006-9084-7)

Google Scholar (http://scholar.google.com/scholar_lookup? title=Continuities\%20and\%20extensions\%20of\%20ethical\%20climate\%20theory\%3A\% 20A\%20meta-

analytic\%2oreview\&author=KD.\%20Martin\&author=JB.\%20Cullen\&journal=Journal\%2 oof\%2oBusiness\%2oEthics\&volume=69\&pages=175-194\&publication_year=2006)

Martin, K. D., Cullen, J. B., Johnson, J. L., \& Parboteeah, K. P. (2007). Decide to bribe: A cross-level analysis of firm and home country influences on bribery activity. Academy of Management Journal, 5O, 1401-1422.

CrossRef (https://doi.org/10.5465/AMJ.2007.28179462)

Google Scholar (http://scholar.google.com/scholar_lookup?

title=Decide\%20to\%2obribe\%3A\%20A\%20cross-

level\%20analysis\%20of\%2ofirm\%20and\%2ohome\%2ocountry\%2oinfluences\%20on\%2 obribery\%20activity\&author=KD.\%20Martin\&author=JB.\%20Cullen\&author=JL.\%2OJ ohnson\&author=KP.\%20Parboteeah\&journal=Academy\%20of\%2oManagement\%20Jou rnal\&volume $=50$ \&pages $=1401-1422 \&$ publication $\_$year $\left.=2007\right)$

Maslow, A. H. (1954). Motivation and personality. New York: Harper.

Google Scholar (http://scholar.google.com/scholar_lookup?

title=Motivation\%20and\%2opersonality\&author=AH.\%20Maslow\&publication_year=19 54)

Mazar, N., Amir, O., \& Ariely, D. (2008). The dishonesty of honest people: A theory of self-concept maintenance. Journal of Marketing Research, 45(6), 633-644.

CrossRef (https://doi.org/10.1509/jmkr.45.6.633)

Google Scholar (http://scholar.google.com/scholar_lookup? title=The\%20dishonesty\%20of\%2ohonest\%2opeople\%3A\%20A\%2otheory\%20of\%20sel f-

concept\%2omaintenance\&author=N.\%20Mazar\&author=0.\%20Amir\&author=D.\%20Ar iely\&journal =Journal\%20of $\% 20$ Marketing $\% 20$ Research $\&$ volume $=45$ \&issue $=6 \&$ pages $=6$ 33-644\&publication_year=2008)

McClelland, D. C. (1967). Money as a motivator: Some research insights. The McKinsey Quarterly, 4, 10-21.

Google Scholar (http://scholar.google.com/scholar_lookup? title=Money\%20as\%20a\%20motivator\%3A\%20Some\%20research\%20insights\&author= DC. $\% 20$ McClelland\&journal $=$ The $\% 20$ McKinsey\%20Quarterly\&volume $=4$ \&pages $=10-$ 21\&publication_year=1967) 
McShane, S. L., \& Von Glinow, M. A. (2008). Organizational behavior (4th ed.). Boston: McGraw-Hill Irwin.

Google Scholar (http://scholar.google.com/scholar_lookup?

title=Organizational\%2obehavior\&author=SL.\%20McShane\&author=MA.\%20Glinow\&p ublication_year=2008)

Merton, R. K. (1968). The Matthew effect in science. Science, 159, 56-63.

CrossRef (https://doi.org/10.1126/science.159.3810.56)

Google Scholar (http://scholar.google.com/scholar_lookup?

title=The\%20Matthew\%20effect\%20in\%20science\&author=RK.\%20Merton\&journal=Sc ience\&volume=159\&pages $=56-63 \&$ publication_year $=1968$ )

Michalos, A. (1985). Multiple discrepancy theory. Social Indicators Research, 16, 347-

413 .

CrossRef (https://doi.org/10.1007/BFoo333288)

Google Scholar (http://scholar.google.com/scholar_lookup?

title=Multiple\%2odiscrepancy\%20theory\&author=A.\%20Michalos\&journal=Social\%2OI ndicators\%20Research\&volume=16\&pages $=347-413 \&$ publication_year $=1985$ )

Mickel, A. E., \& Barron, L. A. (2008). Getting “More Bang for the Buck”: Symbolic value of monetary rewards in organizations. Journal of Management Inquiry, 17, 329-338.

CrossRef (https://doi.org/10.1177/1056492606295502)

Google Scholar (http://scholar.google.com/scholar_lookup?

title=Getting\%20\%E2\%80\%9CMore\%20Bang\%2ofor\%20the\%2oBuck\%E2\%80\%9D\%3

A\%20Symbolic\%20value\%20of\%20monetary\%2orewards\%20in\%2oorganizations\&auth or=AE.\%20Mickel\&author=LA.\%2oBarron\&journal=Journal\%20of\%20Management\%2 oInquiry\&volume=17\&pages=329-338\&publication_year=2008)

Milgram, S. (1974). Obedience to authority: An experimental view. New York: Harper and Row.

Google Scholar (http://scholar.google.com/scholar_lookup?

title=Obedience\%20to\%20authority\%3A\%20An\%20experimental\%20view\&author=S.\% 20Milgram\&publication_year=1974)

Milkovich, G. T., Newman, J. M., \& Gerhart, B. (2014). Compensation (11th ed.). Boston, MA: Irwin/McGraw-Hill.

Google Scholar (http://scholar.google.com/scholar_lookup?

title=Compensation\&author=GT.\%20Milkovich\&author=JM.\%20Newman\&author $=$ B.\% 20Gerhart\&publication_year=2014)

Mitchell, T. R., \& Mickel, A. E. (1999). The meaning of money: An individual difference perspective. Academy of Management Review, 24, 568-578.

Google Scholar (http://scholar.google.com/scholar_lookup? title=The\%20meaning\%20of\%20money\%3A\%20An\%2oindividual\%2odifference\%2oper spective\&author=TR.\%20Mitchell\&author=AE.\%20Mickel\&journal=Academy\%20of\%2 oManagement\%20Review\&volume=24\&pages $=568-578 \&$ publication_year $=1999$ )

Newman, J. M., Gerhart, B., \& Milkovich, G. T. (2017). Compensation (12th ed.). New York: McGraw-Hill.

Google Scholar (https://scholar.google.com/scholar?

q=Newman\%2C\%20J.\%20M.\%2C\%20Gerhart\%2C\%2oB.\%2C\%20\%26\%20Milkovich\%2 C\%20G.\%20T.\%20\%282017\%29.\%20Compensation\%20\%2812th\%2oed.\%29.\%20New\% 20York\%3A\%2oMcGraw-Hill.) 
Nickerson, J. A., \& Zenger, T. R. (2008). Envy, comparison costs, and economic theory of the firm. Strategic Management Journal, 29(13), 1429-1449.

CrossRef (https://doi.org/10.1002/smj.718)

Google Scholar (http://scholar.google.com/scholar_lookup?

title=Envy\%2C\%20comparison\%20costs\%2C\%20and\%2oeconomic\%2otheory\%20of\%2 othe\%2ofirm\&author=JA.\%2oNickerson\&author=TR.\%20Zenger\&journal=Strategic\%2 oManagement\%20Journal\&volume=29\&issue=13\&pages=1429-

1449\&publication_year=2008)

Nkundabanyanga, S. K., Omagor, C., Mpamizo, B., \& Ntayi, J. M. (2011). The love of money, pressure to perform and unethical marketing behavior in the cosmetic industry in Uganda. International Journal of Marketing Studies, 3(4), 40-49.

CrossRef (https://doi.org/10.5539/ijms.v3n4p4o)

Google Scholar (http://scholar.google.com/scholar_lookup?

title=The\%2olove\%20of\%20money\%2C\%2opressure\%20to\%2operform\%20and\%20une thical\%2omarketing\%2obehavior\%20in\%20the\%20cosmetic\%2oindustry\%20in\%2oUga nda\&author=SK.\%20Nkundabanyanga\&author=C.\%20Omagor\&author=B.\%20Mpamiz o\&author=JM.\%20Ntayi\&journal=International\%20Journal\%20of\%2oMarketing\%20St udies\&volume $=3 \&$ issue $=4 \&$ pages $=40-49 \&$ publication $\_$year $=2011$ )

Özbek, M. F., Yoldash, M. A., \& Tang, T. L. P. (2015). Theory of justice, OCB, and individualism: Kyrgyz citizens. Journal of Business Ethics. doi: 10.1007/s10551-0152553-O (https://doi.org/10.1007/s10551-015-2553-0).

Google Scholar (http://scholar.google.com/scholar_lookup? title=Theory\%20of\%20justice\%2C\%20OCB\%2C\%20and\%2oindividualism\%3A\%20 Kyrg yz\%20citizens\&author=MF.\%20\%C3\%96zbek\&author=MA.\%20Yoldash\&author=TLP.\% 20Tang\&journal=Journal\%20of\%20Business\%20Ethics\&publication_year=2015\&doi=1 0.1007\%2Fs10551-015-2553-0)

Pascual-Ezama, D., Fosgaard, T. R., Cardenas, J. C., Kujal, P., Veszteg, R., Gil-Gomez de Liano, B., \& Branas-Garza, P. (2015). Context-dependent cheating: Experimental evidence from 16 countries. Journal of Economic Behavior \& Organization, 116, 379386.

CrossRef (https://doi.org/10.1016/j.jebo.2015.04.020)

Google Scholar (http://scholar.google.com/scholar_lookup?title=Context-

dependent\%20cheating\%3A\%20Experimental\%20evidence\%20from\%2016\%20countrie s\&author=D.\%20Pascual-

Ezama\&author=TR.\%20Fosgaard\&author $=$ JC.\%20Cardenas\&author=P.\%20Kujal\&auth or=R.\%20Veszteg\&author=B.\%20Gil-Gomez\%20de\%2oLiano\&author=P.\%20BranasGarza\&journal=Journal\%20of\%20Economic\%20Behavior\%20\%26\%20Organization\&vo lume=116\&pages=379-386\&publication_year=2015）

Pfeffer, J. (1998, May/June). Six dangerous myths about pay. Harvard Business Review, 108-119.

Google Scholar (https://scholar.google.com/scholar?

q=Pfeffer\%2C\%20J.\%20\%281998\%2C\%20May\%2FJune\%29.\%20Six\%2odangerous\%20 myths\%2Oabout\%2Opay.\%20Harvard\%2OBusiness\%20Review\%2C\%20108\%E2\%80\%9 3119.)

Piff, P. K., Stancato, D. M., Cote, S., \& Mendoza-Denton, R. (2012). Higher social class predicts increased unethical behavior. Proceedings of the National Academy of Sciences of the United States of America, 109, 4086-4091.

CrossRef (https://doi.org/10.1073/pnas.1118373109) 
Google Scholar (http://scholar.google.com/scholar_lookup?

title=Higher\%2osocial\%2oclass\%2opredicts\%2oincreased\%2ounethical\%2obehavior\&a uthor=PK.\%20Piff\&author=DM.\%20Stancato\&author=S.\%20Cote\&author=R.\%20Mend oza-

Denton\&journal=Proceedings\%20of\%20the\%20National\%20Academy\%20of\%20Scienc es\%20of\%20the\%20United\%20States\%20of\%20America\&volume=109\&pages=40864091\&publication_year=2012)

Pinto, J., Leana, C. R., \& Pil, F. K. (2008). Corrupt organizations or organizations of corrupt individuals? Two types of organization-level corruption. Academy of

Management Review, 33, 685-709.

CrossRef (https://doi.org/10.5465/AMR.2008.32465726)

Google Scholar (http://scholar.google.com/scholar_lookup?

title=Corrupt\%20organizations\%200r\%20organizations\%20of\%20corrupt\%2oindividua ls\%3F\%20Two\%2otypes\%20of\%20organization-

level\%20corruption\&author=J.\%20Pinto\&author=CR.\%20Leana\&author=FK.\%20Pil\&j ournal=Academy\%20of\%20Management\%20Review\&volume $=33 \&$ pages $=685$ -

709\&publication_year=2008)

Podsakoff, P. M., MacKenzie, S. B., Lee, J. Y., \& Podsakoff, N. P. (2003). Common method biases in behavioral research: A critical review of the literature and recommended remedies. Journal of Applied Psychology, 88, 879-903.

CrossRef (https://doi.org/10.1037/0021-9010.88.5.879)

Google Scholar (http://scholar.google.com/scholar_lookup?

title=Common\%2omethod\%2obiases\%2oin\%2obehavioral\%2oresearch\%3A\%20A\%20cr itical\%2oreview\%20of\%20the\%2oliterature\%20and\%2orecommended\%2oremedies\&au thor=PM.\%20Podsakoff\&author=SB.\%20MacKenzie\&author=JY.\%2OLee\&author=NP. \%2oPodsakoff\&journal=Journal\%200f\%20Applied\%20Psychology\&volume=88\&pages= 879-903\&publication_year=2003)

Prahalad, C. K., \& Hammond, A. (2002). Serving the world's poor profitably. Harvard Business Review, 80, 48-57.

Google Scholar (http://scholar.google.com/scholar_lookup?

title=Serving\%20the\%20world\%E2\%80\%99s\%20poor\%2oprofitably\&author=CK.\%2OP rahalad\&author=A.\%20Hammond\&journal=Harvard\%20Business\%20Review\&volume= 8o\&pages $=48-57$ \&publication_year $=2002$ )

Report to the Nations (2014). Retrieved October 25, 2015 from http://www.acfe.com/rttn-summary.aspx (http://www.acfe.com/rttn-summary.aspx).

Richman, W. L., Kiesler, S., Weisband, S., \& Drasgow, F. (1999). A meta-analytic study of social desirability distortion in computer-administered questionnaires, traditional questionnaires, and interviews. Journal of Applied Psychology, 84, 754-775.

CrossRef (https://doi.org/10.1037/0021-9010.84.5.754)

Google Scholar (http://scholar.google.com/scholar_lookup?title=A\%2ometaanalytic\%20study\%20of\%20social\%2odesirability\%2odistortion\%2oin\%20computeradministered\%2Oquestionnaires\%2C\%20traditional\%2oquestionnaires\%2C\%20and\%20 interviews\&author $=$ WL.\%20Richman\&author $=$ S.\%20Kiesler\&author $=$ S.\%20Weisband\& author=F.\%20Drasgow\&journal=Journal\%200f\%20Applied\%20Psychology\&volume=84 \&pages $=754-775 \&$ publication_year $=1999)$

Rijekeghem, C. V., \& Weder, B. (1997). Corruption and the rate of temptation: Do low wages in the civil service cause corruption? International Monetary Fund Working Paper, 97/73. 
Google Scholar (https://scholar.google.com/scholar?

q=Rijekeghem\%2C\%20C.\%20V.\%2C\%20\%26\%20Weder\%2C\%2oB.\%20\%281997\%29.\% 20Corruption\%20and\%20the\%2orate\%20of\%2otemptation\%3A\%20Do\%2olow\%20wag es\%20in\%20the\%20civil\%2oservice\%20cause\%20corruption\%3F\%20International\%20 Monetary\%20Fund\%2oWorking\%20Paper\%2C\%2097\%2F73.)

Robinson, S. L., \& Bennett, R. J. (1995). A typology of deviant workplace behaviors: A multidimensional scaling study. Academy of Management Journal, 38, 555-572.

CrossRef (https://doi.org/10.2307/256693)

Google Scholar (http://scholar.google.com/scholar_lookup?

title=A\%20typology\%20of\%2odeviant\%20workplace\%2obehaviors\%3A\%20A\%2omulti dimensional\%20scaling\%20study\&author=SL.\%20Robinson\&author=RJ.\%20Bennett\&j ournal=Academy\%20of\%20Management\%20Journal\&volume=38\&pages=555-

572\&publication_year=1995)

Rousseau, D. M., \& Fried, Y. (2001). Location, location, location: Contextualizing organizational research. Journal of Organizational Behavior, 22, 1-13.

CrossRef (https://doi.org/10.1002/job.78)

Google Scholar (http://scholar.google.com/scholar_lookup?

title=Location\%2C\%2olocation\%2C\%2olocation\%3A\%2oContextualizing\%20organizatio nal\%20research\&author=DM.\%20Rousseau\&author=Y.\%20Fried\&journal=Journal\%20 of\%20Organizational\%2oBehavior\&volume=22\&pages=1-13\&publication_year=2001)

Rynes, S. L., \& Gerhart, B. (2000). Compensation in organizations: Current research and practice. San Francisco, CA: Jossey-Bass.

Google Scholar (http://scholar.google.com/scholar_lookup?

title=Compensation\%20in\%20organizations\%3A\%2oCurrent\%2oresearch\%20and\%2op ractice\&author=SL.\%20Rynes\&author=B.\%20Gerhart\&publication_year=2000)

Sardžoska, E., \& Tang, T. L. P. (2009). Testing a model of behavioral intentions in the Republic of Macedonia: Differences between the private and the public sectors. Journal of Business Ethics, 87, 495-517.

CrossRef (https://doi.org/10.1007/s10551-008-9955-1)

Google Scholar (http://scholar.google.com/scholar_lookup?

title=Testing\%20a\%20model\%20of\%2obehavioral\%2ointentions\%2oin\%20the\%2oRep ublic\%20of\%20Macedonia\%3A\%2oDifferences\%2obetween\%20the\%2oprivate\%20and \%20the\%2opublic\%2osectors\&author=E.\%20Sard\%C5\%BEoska\&author=TLP.\%20Tang \&journal=Journal\%20of\%20Business\%20Ethics\&volume=87\&pages $=495$ -

517\&publication_year=2009)

Sardžoska, E. G., \& Tang, T. L. P. (2012). Work-related behavioral intentions in Macedonia: Coping strategies, work environment, love of money, job satisfaction, and demographic variables. Journal of Business Ethics, 108(3), 373-391.

CrossRef (https://doi.org/10.1007/s10551-011-1096-2)

Google Scholar (http://scholar.google.com/scholar_lookup?title=Workrelated\%20behavioral\%20intentions\%20in\%20Macedonia\%3A\%20Coping\%2ostrategies \%2C\%2Owork\%2Oenvironment\%2C\%2olove\%20of\%2omoney\%2C\%2ojob\%20satisfacti on\%2C\%20and\%20demographic\%20variables\&author=EG.\%20Sard\%C5\%BEoska\&auth or=TLP.\%20Tang\&journal=Journal\%20of\%20Business\%20Ethics\&volume=108\&issue= 3 \&pages $=373-391 \&$ publication_year $=2012)$

Sardžoska, E. G., \& Tang, T. L. P. (2015). Monetary intelligence: Money attitudesunethical intentions, intrinsic and extrinsic job satisfaction, and coping strategies across public and private sectors in Macedonia. Journal of Business Ethics, 13O(1), 93-115. 
CrossRef (https://doi.org/10.1007/s10551-014-2197-5)

Google Scholar (http://scholar.google.com/scholar_lookup?

title=Monetary\%2ointelligence\%3A\%20Money\%20attitudes\%E2\%80\%94unethical\%20i ntentions\%2C\%2ointrinsic\%20and\%20extrinsic\%20job\%20satisfaction\%2C\%20and\%2 ocoping\%20strategies\%20across\%2opublic\%20and\%2oprivate\%2osectors\%20in\%20M acedonia\&author=EG.\%20Sard\%C5\%BEoska\&author=TLP.\%20Tang\&journal=Journal \%20of\%20Business\%20Ethics\&volume $=130 \&$ issue $=1$ \&pages $=93^{-}$

115\&publication_year=2015)

Scandura, T. A. (2016). Essentials of organizational behavior: An evidence-based approach. Los Angeles: Sage.

Google Scholar (http://scholar.google.com/scholar_lookup?

title=Essentials\%20of\%20organizational\%20behavior\%3A\%20An\%20evidence-

based\%20approach\&author=TA.\%20Scandura\&publication_year=2016)

Scherbaum, C. A., \& Ferreter, J. M. (2009). Estimating statistical power and required sample sizes for organizational research using multilevel modeling. Organizational Research Methods, 12, 347-367.

CrossRef (https://doi.org/10.1177/1094428107308906)

Google Scholar (http://scholar.google.com/scholar_lookup?

title=Estimating\%20statistical\%20power\%20and\%2orequired\%20sample\%20sizes\%2of or\%20organizational\%2oresearch\%2ousing\%2omultilevel\%2omodeling\&author=CA.\%2 oScherbaum\&author=JM.\%20Ferreter\&journal=Organizational\%20Research\%20Metho ds\&volume=12\&pages=347-367\&publication_year $=2009)$

Schoorman, F. D., \& Mayer, R. C. (2008). The value of common perspectives in selfreported appraisals: You get what you ask for. Organizational Research Methods, 11, 148-159.

CrossRef (https://doi.org/10.1177/1094428107307168)

Google Scholar (http://scholar.google.com/scholar_lookup?

title=The\%20value\%20of\%20common\%2operspectives\%20in\%2oself-

reported\%20appraisals\%3A\%20You\%20get\%20what\%20you\%20ask\%2ofor\&author=F

D.\%20Schoorman\&author=RC.\%20Mayer\&journal=Organizational\%20Research\%20Me thods\&volume=11\&pages $=148-159$ \&publication_year=2008)

Simons, T. (2002). Behavioral integrity: The perceived alignment between managers' words and deeds as a research focus. Organization Science, 13, 18-35.

CrossRef (https://doi.org/10.1287/orsc.13.1.18.543)

Google Scholar (http://scholar.google.com/scholar_lookup?

title=Behavioral\%20integrity\%3A\%20The\%2operceived\%20alignment\%2obetween\%20 managers\%E2\%80\%99\%20words\%20and\%2odeeds\%20as\%20a\%2oresearch\%2ofocus\& author $=\mathrm{T} . \% 20$ Simons\&journal $=$ Organization $\% 20$ Science $\&$ volume $=13 \&$ pages $=18$ -

35\&publication_year=2002)

Simons, T., \& Roberson, Q. (2003). Why managers should care about fairness: The effects of aggregate justice perceptions on organizational outcomes. Journal of Applied Psychology, 88, 432-443.

CrossRef (https://doi.org/10.1037/0021-9010.88.3.432)

Google Scholar (http://scholar.google.com/scholar_lookup?

title=Why\%20managers\%20should\%20care\%20about\%2ofairness\%3A\%2oThe\%2oeffec ts\%20of\%20aggregate\%2ojustice\%2operceptions\%20on\%2oorganizational\%20outcome s\&author=T.\%20Simons\&author=Q.\%20Roberson\&journal=Journal\%20of\%20Applied \%20Psychology\&volume $=88 \&$ pages $=432-443 \&$ publication_year $=2003$ ) 
Skarlicki, D. P., \& Folger, R. (1997). Retaliation in the workplace: The roles of distributive, procedural, and interactional justice. Journal of Applied Psychology, 82, 434-443.

CrossRef (https://doi.org/10.1037/0021-9010.82.3.434)

Google Scholar (http://scholar.google.com/scholar_lookup?

title=Retaliation\%20in\%20the\%20workplace\%3A\%20The\%2oroles\%20of\%2odistributiv e\%2C\%2oprocedural\%2C\%20and\%20interactional\%20justice\&author=DP.\%20Skarlicki \&author=R.\%20Folger\&journal=Journal\%20of\%20Applied\%20Psychology\&volume=82 \&pages=434-443\&publication_year=1997)

Skinner, B. F. (1972). Beyond freedom and dignity. New York: Bantam Books.

Google Scholar (http://scholar.google.com/scholar_lookup?

title=Beyond\%2ofreedom\%20and\%20dignity\&author=BF.\%2oSkinner\&publication_yea $\mathrm{r}=1972$ )

Smith, A. (1937). An inquiry into the nature and causes of the wealth of nations. New York: Modern Library. (Original work published 1776).

Google Scholar (http://scholar.google.com/scholar_lookup?

title=An\%20inquiry\%20into\%20the\%20nature\%20and\%20causes\%20of\%20the\%20we alth\%20of\%2onations\&author=A.\%20Smith\&publication_year=1937)

Sorensen, J. B. (2002). The strength of corporate culture and the reliability of firm performance. Administrative Science Quarterly, 47, 70-91.

CrossRef (https://doi.org/10.2307/3094891)

Google Scholar (http://scholar.google.com/scholar_lookup? title=The\%20strength\%20of\%20corporate\%20culture\%20and\%20the\%2oreliability\%20 of\%20firm\%2operformance\&author=JB.\%20Sorensen\&journal=Administrative\%20Scie nce\%20Quarterly\&volume=47\&pages $=70-91 \&$ publication_year $=2002$ )

Spector, P. E. (2006). Method variance in organizational research: Truth or urban legend? Organizational Research Methods, 9, 221-232.

CrossRef (https://doi.org/10.1177/1094428105284955)

Google Scholar (http://scholar.google.com/scholar_lookup?

title=Method\%2ovariance\%20in\%20organizational\%2oresearch\%3A\%20Truth\%20or\% 2ourban\%2olegend\%3F\&author=PE.\%20Spector\&journal=Organizational\%2oResearch \%20Methods\&volume=9\&pages $=221-232 \&$ publication_year=2006)

Spector, P. E., \& Fox, S. (2010). Counterproductive work behavior and organizational citizenship behavior: Are they opposite forms of active behavior? Applied Psychology: An International Review, 59(1), 21-39.

CrossRef (https://doi.org/10.1111/j.1464-0597.2009.00414.x)

Google Scholar (http://scholar.google.com/scholar_lookup?

title=Counterproductive\%20work\%2obehavior\%20and\%20organizational\%2ocitizenshi p\%2obehavior\%3A\%2oAre\%2othey\%20opposite\%2oforms\%20of\%2oactive\%2obehavio r\%3F\&author=PE.\%20Spector\&author=S.\%20Fox\&journal=Applied\%20Psychology\%3A \%20An\%20International\%20Review\&volume $=59$ \&issue $=1$ \&pages $=21$ -

39\&publication_year=2010)

Srivastava, A., Locke, E. A., \& Bartol, K. M. (2001). Money and subjective well-being: It's not the money, it's the motives. Journal of Personality and Social Psychology, 80, 959971.

CrossRef (https://doi.org/10.1037/0022-3514.80.6.959)

Google Scholar (http://scholar.google.com/scholar_lookup?

title=Money\%20and\%20subjective\%20well- 
being\%3A\%20It\%E2\%80\%99s\%20not\%20the\%2omoney\%2C\%2oit\%E2\%80\%99s\%20t he\%2omotives\&author=A.\%20Srivastava\&author=EA.\%2oLocke\&author=KM.\%20Bart ol\&journal=Journal\%20of\%2oPersonality\%20and\%20Social\%20Psychology\&volume=8 o\&pages=959-971\&publication_year $=2001$ )

Staw, B. M., Bell, N. E., \& Clausen, J. A. (1986). The dispositional approach to job attitudes: A lifetime longitudinal test. Administrative Science Quarterly, 31, 56-77. CrossRef (https://doi.org/10.2307/2392766)

Google Scholar (http://scholar.google.com/scholar_lookup?

title=The\%20dispositional\%20approach\%20to\%20job\%20attitudes\%3A\%20A\%2olifeti me\%2olongitudinal\%20test\&author=BM.\%20Staw\&author=NE.\%20Bell\&author=JA.\% 20Clausen\&journal=Administrative $\% 20$ Science $\% 20$ Quarterly\&volume $=31$ \&pages $=56$ 77\&publication_year=1986)

Tang, T. L. P. (1992). The meaning of money revisited. Journal of Organizational Behavior, 13, 197-202.

CrossRef (https://doi.org/10.1002/job.4030130209)

Google Scholar (http://scholar.google.com/scholar_lookup?

title=The\%20meaning\%20of\%20money\%20revisited\&author=TLP.\%20Tang\&journal=J ournal\%20of\%20Organizational\%2oBehavior\&volume=13\&pages=197-

202\&publication_year=1992)

Tang, T. L. P. (1993). The meaning of money: Extension and exploration of the money ethic scale in a sample of university students in Taiwan. Journal of Organizational

Behavior, 14, 93-99.

CrossRef (https://doi.org/10.1002/job.4030140109)

Google Scholar (http://scholar.google.com/scholar_lookup?

title=The\%20meaning\%20of\%20money\%3A\%20Extension\%20and\%2oexploration\%20 of\%20the\%20money\%20ethic\%20scale\%20in\%20a\%20sample\%20of\%20university\%20 students\%20in\%20Taiwan\&author=TLP.\%20Tang\&journal=Journal\%20of\%2oOrganiza tional\%2oBehavior\&volume=14\&pages=93-99\&publication_year=1993）

Tang, T. L. P. (1996). Pay differentials as a function of rater's sex, money ethic, and job incumbent's sex: A test of the Matthew Effect. Journal of Economic Psychology, 17, 127144 .

CrossRef (https://doi.org/10.1016/0167-4870(95)00038-0)

Google Scholar (http://scholar.google.com/scholar_lookup?

title=Pay\%2odifferentials\%20as\%20a\%20function\%20of\%20rater\%E2\%80\%99s\%20sex \%2C\%2omoney\%2oethic\%2C\%20and\%20job\%2oincumbent\%E2\%80\%99s\%20sex\%3A

\%20A\%20test\%20of\%20the\%2oMatthew\%20Effect\&author=TLP.\%20Tang\&journal=Jo urnal\%20of\%20Economic\%20Psychology\&volume=17\&pages $=127-$

144\&publication_year=1996)

Tang, T. L. P. (2010). Money, the meaning of money, management, spirituality, and religion. Journal of Management, Spirituality and Religion, 7, 173-189.

CrossRef (https://doi.org/10.1080/14766081003746448)

Google Scholar (http://scholar.google.com/scholar_lookup?

title=Money\%2C\%2othe\%20meaning\%20of\%2omoney\%2C\%2omanagement\%2C\%20sp irituality\%2C\%20and\%2oreligion\&author=TLP.\%20Tang\&journal=Journal\%20of\%20M anagement\%2C\%20Spirituality\%20and\%20Religion\&volume $=7$ \&pages $=173-$

189\&publication_year=2010)

Tang, T. L. P. (2014). Theory of monetary intelligence: Money attitudes-religious values, making money, making ethical decisions, and making the grade. Journal of Business 
Ethics. doi: 10.1007/s10551-014-2411-5 (https://doi.org/10.1007/s10551-014-2411-5). Google Scholar (http://scholar.google.com/scholar_lookup? title=Theory\%20of\%2omonetary\%20intelligence\%3A\%20Money\%20attitudes\%E2\%80 \%94religious\%20values\%2C\%2omaking\%20money\%2C\%20making\%2oethical\%2odecis ions\%2C\%20and\%2omaking\%20the\%2ograde\&author=TLP.\%20Tang\&journal=Journal \%20of\%20Business\%20Ethics\&publication_year=2014\&doi=10.1007\%2Fs10551-0142411-5)

Tang, T. L. P., \& Baumeister, R. F. (1984). Effects of personal values, perceived surveillance, and task labels on task preference: The ideology of turning play into work. Journal of Applied Psychology, 69, 99-105.

CrossRef (https://doi.org/10.1037/0021-9010.69.1.99)

Google Scholar (http://scholar.google.com/scholar_lookup? title=Effects\%20of\%2opersonal\%20values\%2C\%2operceived\%2osurveillance\%2C\%20a nd\%20task\%2olabels\%20on\%2otask\%2opreference\%3A\%20The\%2oideology\%20of\%20 turning\%20play\%20into\%20work\&author=TLP.\%20Tang\&author=RF.\%2oBaumeister\& journal=Journal\%20of\%20Applied\%20Psychology\&volume=69\&pages=99105\&publication_year=1984)

Tang, T. L. P., \& Chen, Y. J. (2008). Intelligence vs. wisdom: The love of money, Machiavellianism, and unethical behavior across college major and gender. Journal of Business Ethics, 82, 1-26.

CrossRef (https://doi.org/10.1007/s10551-007-9559-1)

Google Scholar (http://scholar.google.com/scholar_lookup? title=Intelligence\%20vs.\%20wisdom\%3A\%20The\%2olove\%20of\%2omoney\%2C\%20Ma chiavellianism\%2C\%20and\%2ounethical\%2obehavior\%20across\%20college\%2omajor\% 20and\%2ogender\&author=TLP.\%20Tang\&author=YJ.\%20Chen\&journal=Journal\%20of \%20Business\%20Ethics\&volume=82\&pages=1-26\&publication_year=2008)

Tang, T. L. P., Chen, Y. J., \& Sutarso, T. (2008a). Bad apples in bad (business) barrels: The love of money, Machiavellianism, risk tolerance, and unethical behavior.

Management Decision, 46(2), 243-263.

CrossRef (https://doi.org/10.1108/00251740810854140)

Google Scholar (http://scholar.google.com/scholar_lookup?

title=Bad\%20apples\%20in\%2obad\%20\%28business\%29\%2obarrels\%3A\%2oThe\%2olov e\%20of\%20money\%2C\%20Machiavellianism\%2C\%2orisk\%20tolerance\%2C\%20and\%2 ounethical\%2obehavior\&author=TLP.\%20Tang\&author=YJ.\%20Chen\&author=T.\%2OS utarso\&journal $=$ Management\%20Decision \&volume $=46 \&$ issue $=2 \&$ pages $=243-$ 263\&publication_year $=2008$ )

Tang, T. L. P., \& Chiu, R. K. (2003). Income, Money Ethic, pay satisfaction, commitment, and unethical behavior: Is the love of money the root of evil for Hong Kong managers? Journal of Business Ethics, 46, 13-30.

CrossRef (https://doi.org/10.1023/A\%3A1024731611490)

Google Scholar (http://scholar.google.com/scholar_lookup? title=Income\%2C\%20Money\%20Ethic\%2C\%2opay\%20satisfaction\%2C\%20commitmen t\%2C\%20and\%2ounethical\%2obehavior\%3A\%2oIs\%20the\%2olove\%20of\%2omoney\%2 othe\%2oroot\%20of\%20evil\%2ofor\%20Hong\%20Kong\%20managers\%3F\&author=TLP. \%20Tang\&author=RK.\%20Chiu\&journal=Journal\%20of\%2oBusiness\%20Ethics\&volum $\mathrm{e}=46$ \&pages $=13-30$ \&publication_year=2003)

Tang, T. L. P., Kim, J. K., \& Tang, D. S. H. (2000). Does attitude toward money moderate the relationship between intrinsic job satisfaction and voluntary turnover? Human 
Relations, 53, 213-245.

CrossRef (https://doi.org/10.1177/a010560)

Google Scholar (http://scholar.google.com/scholar_lookup?

title=Does\%20attitude\%20toward\%20money\%20moderate\%20the\%2orelationship\%20 between\%2ointrinsic\%20job\%20satisfaction\%20and\%20voluntary\%20turnover\%3F\&au thor=TLP.\%20Tang\&author=JK.\%20Kim\&author=DSH.\%20Tang\&journal=Human\%20 Relations\&volume=53\&pages $=213-245 \&$ publication_year=2000)

Tang, T. L. P., \& Liu, H. (2012). Love of money and unethical behavior intention: Does an authentic supervisor's personal integrity and character (ASPIRE) make a difference?

Journal of Business Ethics, 107(3), 295-312.

CrossRef (https://doi.org/10.1007/s10551-011-1040-5)

Google Scholar (http://scholar.google.com/scholar_lookup?

title=Love\%20of\%20money\%20and\%2ounethical\%2obehavior\%2ointention\%3A\%2oDo es\%20an\%20authentic\%20supervisor\%E2\%80\%99s\%2opersonal\%2ointegrity\%20and\% 20character\%20\%28ASPIRE\%29\%20make\%20a\%2odifference\%3F\&author=TLP.\%20T ang\&author=H.\%20Liu\&journal=Journal\%20of\%20Business\%20Ethics\&volume=107\&i ssue $=3 \&$ pages $=295-312 \&$ publication_year $=2012)$

Tang, T. L. P., Luna-Arocas, R., Quintanilla Pardo, I., \& Tang, T. L. N. (2014). Materialism and the bright and dark sides of the financial dream in Spain: The positive role of money attitudes-The Matthew Effect. Applied Psychology: An International Review, 63(3), 480-508.

CrossRef (https://doi.org/10.1111/j.1464-0597.2012.00533.x)

Google Scholar (http://scholar.google.com/scholar_lookup?

title=Materialism\%20and\%20the\%2obright\%20and\%20dark\%2osides\%20of\%20the\%2 ofinancial\%20dream\%20in\%20Spain\%3A\%20The\%20positive\%20role\%20of\%20money \%20attitudes\%E2\%80\%94The\%20Matthew\%2oEffect\&author=TLP.\%20Tang\&author= R.\%20Luna-

Arocas\&author=I.\%20Quintanilla\%20Pardo\&author=TLN.\%20Tang\&journal=Applied\% 20Psychology\%3A\%20An\%20International\%20Review\&volume $=63 \&$ \&issue $=3$ \&pages $=48$ 0-508\&publication_year=2014)

Tang, T. L. P., Luna-Arocas, R., \& Sutarso, T. (2005). From income to pay satisfaction: The love of money and pay equity comparison as mediators and culture (the United States and Spain) and gender as moderators. Management Research: The Journal of the Iberoamerican Academy of Management, 3, 7-26.

Google Scholar (http://scholar.google.com/scholar_lookup? title=From\%20income\%20to\%20pay\%20satisfaction\%3A\%20The\%2olove\%20of\%20mo ney\%20and\%20pay\%20equity\%20comparison\%20as\%2omediators\%20and\%2oculture \%20\%28the\%2oUnited\%2oStates\%20and\%20Spain\%29\%20and\%2ogender\%20as\%20 moderators\&author=TLP.\%20Tang\&author=R.\%20Luna-

Arocas\&author=T.\%20Sutarso\&journal=Management\%20Research\%3A\%20The\%20Jou rnal\%20of\%2othe\%2oIberoamerican\%20Academy\%20of\%20Management\&volume=3\& pages $=7-26 \&$ publication_year $=2005)$

Tang, T. L. P., \& Sutarso, T. (2013). Falling or not falling into temptation? Multiple faces of temptation, monetary intelligence, and unethical intentions across gender. Journal of Business Ethics, 116(3), 529-552.

CrossRef (https://doi.org/10.1007/s10551-012-1475-3)

Google Scholar (http://scholar.google.com/scholar_lookup?

title=Falling\%20or\%2onot\%2ofalling\%2ointo\%2otemptation\%3F\%20Multiple\%2ofaces 
\%20of\%20temptation\%2C\%2omonetary\%2ointelligence\%2C\%20and\%2ounethical\%20i ntentions\%20across\%20gender\&author=TLP.\%20Tang\&author=T.\%20Sutarso\&journal $=$ Journal\%20of $\% 20$ Business $\% 20$ Ethics $\&$ volume $=116 \&$ issue $=3$ \&pages $=529$ -

552\&publication_year=2013)

Tang, T. L. P., Sutarso, T., Akande, A., Allen, M. W., Alzubaidi, A. S., Ansari, M. A. et al. (2006a). The love of money and pay level satisfaction: Measurement and functional equivalence in 29 geographical entities around the world. Management and Organization Review, 2, 423-452.

CrossRef (https://doi.org/10.1111/j.1740-8784.2006.00051.x) Google Scholar (http://scholar.google.com/scholar_lookup?

title=The\%2olove\%20of\%20money\%20and\%20pay\%2olevel\%2osatisfaction\%3A\%2oM easurement\%20and\%20functional\%20equivalence\%20in\%2029\%2ogeographical\%20en tities\%20around\%20the\%20world\&author=TLP.\%20Tang\&author=T.\%20Sutarso\&auth or=A.\%20Akande\&author=MW.\%20Allen\&author=AS.\%20Alzubaidi\&author=MA.\%20 Ansari\&journal=Management\%20and\%20Organization\%20Review\&volume=2\&pages= 423-452\&publication_year=2006)

Tang, T. L. P., Sutarso, T., Ansari, M. A., Lim, V. K. G., Teo, T. S. H., Arias-Galicai, F. et al. (2011). The love of money is the root of all evil: Pay satisfaction and CPI as moderators. Paper presented at the Academy of Management Annual Meeting, San Antonio, TX, 12-16 August 2011. In L. A. Toombs (Ed.), Best paper proceedings of the 2011 Academy of Management.

Google Scholar (https://scholar.google.com/scholar? q=Tang\%2C\%20T.\%2OL.\%2OP.\%2C\%2oSutarso\%2C\%2OT.\%2C\%2OAnsari\%2C\%2OM.\% 20A.\%2C\%2OLim\%2C\%2OV.\%2OK.\%2OG.\%2C\%2OTeo\%2C\%2OT.\%2OS.\%2OH.\%2C\%2O Arias-

Galicai\%2C\%2oF.\%20et\%20al.\%20\%282011\%29.\%2oThe\%2olove\%20of\%2omoney\%20 is\%2othe\%2oroot\%20of\%20all\%20evil\%3A\%20Pay\%2osatisfaction\%20and\%20CPI\%20 as\%20moderators.\%20Paper\%2opresented\%20at\%20the\%20Academy\%20of\%20Mana gement\%2OAnnual\%2oMeeting\%2C\%2OSan\%2OAntonio\%2C\%2OTX\%2C\%2012\%E2\%8 0\%9316\%20August\%202011.\%2OIn\%2OL.\%20A.\%20Toombs\%20\%28Ed.\%29\%2C\%20B est\%20paper\%2oproceedings\%20of\%20the\%202011\%20Academy\%20of\%20Manageme nt.\%oA\%20\%20\%20\%20\%20\%20\%20\%20\%20\%20\%20\%20\%20\%20\%20\%20)

Tang, T. L. P., Sutarso, T., Ansari, M. A., Lim, V. K. G., Teo, T. S. H., Arias-Galicai, F. et al. (2013). Are money smart people satisfied with pay and life? A theory of monetary intelligence. Paper presented at Academy of Management Annual Meeting, Lake Buena Vista (Orlando), Florida.

Google Scholar (https://scholar.google.com/scholar? q=Tang\%2C\%20T.\%20L.\%20P.\%2C\%2oSutarso\%2C\%20T.\%2C\%20Ansari\%2C\%20M.\% 20A.\%2C\%2OLim\%2C\%2OV.\%2OK.\%2OG.\%2C\%2OTeo\%2C\%2OT.\%2oS.\%2OH.\%2C\%2O Arias-

Galicai\%2C\%20F.\%20et\%20al.\%20\%282013\%29.\%20Are\%20money\%20smart\%2opeop le\%20satisfied\%20with\%2opay\%20and\%2olife\%3F\%20A\%2otheory\%20of\%2omonetar y\%2ointelligence.\%20Paper\%2opresented\%20at\%20Academy\%20of\%20Management\% 20Annual\%2OMeeting\%2C\%2oLake\%2oBuena\%2OVista\%20\%28Orlando\%29\%2C\%2OF lorida.)

Tang, T. L. P., Sutarso, T., Ansari, M. A., Lim, V. K. G., Teo, T. S. H., Arias-Galicai, F. et al. (2015). Monetary intelligence and behavioral economics across 32 cultures: Good 
apples enjoy good quality of life in good barrels. Journal of Business Ethics.

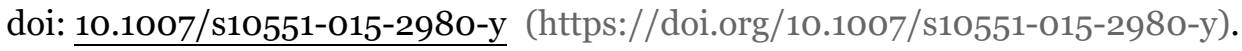

Tang, T. L. P., Sutarso, T., Davis, G. M. T., Dolinski, D., Ibrahim, A. H. S., \& Wagner, S. L. (2008b). To help or not to help? The Good Samaritan Effect and the love of money on helping behavior. Journal of Business Ethics, 82(4), 865-887.

CrossRef (https://doi.org/10.1007/s10551-007-9598-7)

Google Scholar (http://scholar.google.com/scholar_lookup?

title=To\%2ohelp\%20or\%20not\%20to\%2ohelp\%3F\%20The\%2oGood\%20Samaritan\%20 Effect\%20and\%20the\%2olove\%20of\%20money\%20on\%2ohelping\%2obehavior\&author $=$ TLP.\%20Tang\&author $=\mathrm{T} . \% 20$ Sutarso\&author $=$ GMT.\%20Davis\&author $=$ D.\%20Dolins ki\&author=AHS.\%2OIbrahim\&author=SL.\%20Wagner\&journal=Journal\%20of\%2oBusi ness $\% 20$ Ethics\&volume $=82$ \&issue $=4 \&$ pages $=865-887$ \&publication_year $=2008$ )

Tang, T. L. P., \& Tang, T. L. N. (2010). Finding the lost sheep: A panel study of business students' intrinsic religiosity, Machiavellianism, and unethical behavior intention in a public institution. Ethics and Behavior, 2O(5), 1-28.

CrossRef (https://doi.org/10.1080/10508422.2010.491763)

Google Scholar (http://scholar.google.com/scholar_lookup?

title=Finding\%20the\%20lost\%20sheep\%3A\%20A\%20panel\%2ostudy\%20of\%2obusines s\%20students\%E2\%80\%99\%20intrinsic\%20religiosity\%2C\%20Machiavellianism\%2C\% 20and\%20unethical\%2obehavior\%2ointention\%20in\%20a\%2opublic\%2oinstitution\&au thor=TLP.\%20Tang\&author=TLN.\%20Tang\&journal=Ethics\%20and\%2oBehavior\&volu $\mathrm{me}=20 \&$ issue $=5 \&$ pages $=1-28 \&$ publication_year $=2010$ )

Tang, T. L. P., Tang, T. L. N., \& Homaifar, B. Y. (2006b). Income, the love of money, pay comparison, and pay satisfaction: Race and gender as moderators. Journal of

Managerial Psychology, 21, 476-491.

CrossRef (https://doi.org/10.1108/02683940610673988)

Google Scholar (http://scholar.google.com/scholar_lookup?

title=Income\%2C\%20the\%2olove\%20of\%2omoney\%2C\%2opay\%2ocomparison\%2C\%2 oand\%2opay\%20satisfaction\%3A\%20Race\%20and\%2ogender\%20as\%2omoderators\&a uthor=TLP.\%20Tang\&author=TLN.\%20Tang\&author=BY.\%20Homaifar\&journal=Jour nal\%20of\%2oManagerial\%20Psychology\&volume $=21 \&$ pages $=476-$

491\&publication_year=2006)

Tepper, B. J., Carr, J. C., Breaux, D. M., Geider, S., Hu, C. Y., \& Hua, W. (2009). Abusive supervision, intentions to quit, and employees' workplace deviance: A

power/dependence analysis. Organizational Behavior and Human Decision Processes, 109, 156-167.

CrossRef (https://doi.org/10.1016/j.obhdp.2009.03.004)

Google Scholar (http://scholar.google.com/scholar_lookup?

title=Abusive\%20supervision\%2C\%2ointentions\%2oto\%2Oquit\%2C\%20and\%20employ ees\%E2\%80\%99\%2Oworkplace\%2Odeviance\%3A\%20A\%2opower\%2Fdependence\%20a nalysis\&author=BJ.\%20Tepper\&author=JC.\%20Carr\&author=DM.\%20Breaux\&author= S.\%20Geider\&author=CY.\%20Hu\&author $=\mathrm{W} . \% 20 H u a \& j o u r n a l=O r g a n i z a t i o n a l \% 20 B e h$ avior\%20and\%20Human\%20Decision\%20Processes\&volume=109\&pages=156-

167\&publication_year=2009)

Tepper, B. J., Moss, S. E., Lockhart, D. E., \& Carr, J. C. (2007). Abusive supervision, upward maintenance communication, and subordinates' psychological distress.

Academy of Management Journal, 13, 197-202. 
Google Scholar (http://scholar.google.com/scholar_lookup?

title=Abusive $\%$ 2Osupervision\%2C\%2oupward\%2omaintenance\%2ocommunication\%2C \%20and\%20subordinates\%E2\%80\%99\%20psychological\%20distress\&author=BJ.\%20T epper\&author=SE.\%20Moss\&author=DE.\%20Lockhart\&author=JC.\%20Carr\&journal= Academy\%20of\%20Management\%20Journal\&volume=13\&pages=197-

202\&publication_year=2007)

Treisman, D. (2007). What have we learned about the causes of corruption from ten years of cross-national empirical research? Annual Review of Political Science, 10, 211244.

CrossRef (https://doi.org/10.1146/annurev.polisci.10.081205.095418)

Google Scholar (http://scholar.google.com/scholar_lookup?

title=What\%20have\%20we\%2olearned\%20about\%20the\%20causes\%20of\%20corruptio n\%2ofrom\%2oten\%20years\%20of\%20cross-

national\%20empirical\%2oresearch\%3F\&author=D.\%20Treisman\&journal=Annual\%20 Review\%20of\%20Political\%20Science\&volume=10\&pages $=211-$

244\&publication_year=2007)

Treviño, L. K. (1986). Ethical decision-making in organizations: A person-situation interactionist model. Academy of Management Review, 11, 601-617.

Google Scholar (http://scholar.google.com/scholar_lookup?title=Ethical\%2odecisionmaking\%20in\%20organizations\%3A\%20A\%2operson-

situation\%2ointeractionist\%2omodel\&author=LK.\%20Trevi\%C3\%B1o\&journal=Academ y\%20of\%20Management\%20Review\&volume=11\&pages=601-

617\&publication_year=1986)

Treviño, L. K., \& Brown, M. E. (2004). Managing to be ethical: Debunking five business ethics myths. Academy of Management Executive, 18(2), 69-81.

CrossRef (https://doi.org/10.5465/AME.2004.13837400)

Google Scholar (http://scholar.google.com/scholar_lookup?

title=Managing\%20to\%2obe\%2oethical\%3A\%2oDebunking\%2ofive\%2obusiness\%2oeth ics\%20myths\&author=LK.\%20Trevi\%C3\%B1o\&author=ME.\%20Brown\&journal=Acade my\%20of\%2oManagement\%2oExecutive\&volume=18\&issue=2\&pages=69-

81\&publication_year=2004)

Treviño, L. K., Hartman, L. P., \& Brown, M. (2000). Moral person and moral manager: How executives develop a reputation for ethical leadership. California Management Review, 42(4), 128-142.

CrossRef (https://doi.org/10.2307/41166057)

Google Scholar (http://scholar.google.com/scholar_lookup?

title=Moral\%20person\%20and\%20moral\%2omanager\%3A\%20How\%2oexecutives\%20 develop\%20a\%2oreputation\%2ofor\%20ethical\%2oleadership\&author=LK.\%20Trevi\%C 3\%B1o\&author=LP.\%20Hartman\&author=M.\%20Brown\&journal=California\%2OManag ement\%20Review\&volume $=42 \&$ issue $=4$ \&pages $=128-142 \&$ publication_year $=2000$ )

Vandenberg, R. J., \& Lance, C. E. (2000). A review and synthesis of the measurement invariance literature: Suggestions, practices, and recommendations for organizational research. Organizational Research Methods, 3, 4-69.

CrossRef (https://doi.org/10.1177/109442810031002)

Google Scholar (http://scholar.google.com/scholar_lookup?

title=A\%20review\%20and\%20synthesis\%20of\%20the\%20measurement\%2oinvariance \%2oliterature\%3A\%20Suggestions\%2C\%2opractices\%2C\%20and\%2orecommendations \%2ofor\%20organizational\%2oresearch\&author=RJ.\%20Vandenberg\&author=CE.\%2OL 
ance\&journal=Organizational\%20Research\%2oMethods\&volume=3\&pages=4-

69\&publication_year=2000)

Vardi, Y., \& Weitz, E. (2004). Misbehavior in organizations. Mahwah, NY: Lawrence

Erlbaum.

Google Scholar (http://scholar.google.com/scholar_lookup?

title=Misbehavior\%20in\%20organizations\&author=Y.\%20Vardi\&author=E.\%20Weitz\&

publication_year=2004)

Victor, B., \& Cullen, J. B. (1988). The organizational bases of ethical work climates.

Administrative Science Quarterly, 33, 101-125.

CrossRef (https://doi.org/10.2307/2392857)

Google Scholar (http://scholar.google.com/scholar_lookup?

title=The\%20organizational\%2obases\%20of\%20ethical\%20work\%2oclimates\&author=

B.\%20Victor\&author=JB.\%20Cullen\&journal=Administrative\%20Science\%20Quarterly

\&volume $=33 \&$ pages $=101-125 \&$ publication_year $=1988$ )

Vitell, S. J., \& Hidalgo, E. R. (2006). The impact of corporate ethical values and enforcement of ethical codes on the perceived importance of ethics in business: A comparison of US and Spanish managers. Journal of Business Ethics, 64(1), 31-43.

CrossRef (https://doi.org/10.1007/s10551-005-4664-5)

Google Scholar (http://scholar.google.com/scholar_lookup?

title=The\%20impact\%20of\%20corporate\%20ethical\%20values\%20and\%2oenforcement \%20of\%20ethical\%20codes\%20on\%20the\%2operceived\%2oimportance\%20of\%20ethic s\%20in\%2obusiness\%3A\%20A\%20comparison\%20of\%2oUS\%20and\%2oSpanish\%2om anagers\&author=SJ.\%20Vitell\&author=ER.\%20Hidalgo\&journal=Journal\%20of\%20Bus iness\%20Ethics\&volume $=64$ \&issue $=1 \&$ pages $=31-43$ \&publication $\_$year $=2006$ )

Vitell, S. J., Paolillo, J. G. P., \& Singh, J. J. (2006). The role of money and religiosity in determining consumers' ethical beliefs. Journal of Business Ethics, 64, 117-124.

CrossRef (https://doi.org/10.1007/s10551-005-1901-x)

Google Scholar (http://scholar.google.com/scholar_lookup?

title=The\%20role\%20of\%2omoney\%20and\%20religiosity\%2oin\%2odetermining\%20co

nsumers\%E2\%80\%99\%20ethical\%2obeliefs\&author=SJ.\%20Vitell\&author=JGP.\%20Pa olillo\&author=JJ.\%20Singh\&journal=Journal\%20of\%20Business\%20Ethics\&volume $=6$ 4\&pages $=117-124 \&$ publication_year $=2006$ )

Vohs, K. D., Mead, N. L., \& Goode, M. (2006). The psychological consequences of money. Science, 314, 1154-1156.

CrossRef (https://doi.org/10.1126/science.1132491)

Google Scholar (http://scholar.google.com/scholar_lookup?

title=The\%20psychological\%20consequences\%20of\%20money\&author=KD.\%20Vohs\&a uthor=NL.\%20Mead\&author=M.\%20Goode\&journal $=$ Science\&volume=314\&pages $=115$ 4-1156\&publication_year=2006)

Vynoslavaska, O., McKinney, J. A., Moore, C. W., \& Longenecker, J. G. (2005).

Transition ethics: A comparison of Ukrainian and United States business professionals.

Journal of Business Ethics, 61, 283-299.

CrossRef (https://doi.org/10.1007/s10551-005-1128-x)

Google Scholar (http://scholar.google.com/scholar_lookup?

title=Transition\%2oethics\%3A\%20A\%20comparison\%20of\%2oUkrainian\%20and\%20U nited\%20States\%2obusiness\%20professionals\&author=0.\%20Vynoslavaska\&author=JA \%20McKinney\&author=CW.\%20Moore\&author=JG.\%20Longenecker\&journal=Journal \%20of\%20Business\%20Ethics\&volume $=61 \&$ pages $=283-299 \&$ publication_year $=2005$ ) 
Welsh, D. T., \& Ordóñez, L. D. (2014). Conscience without cognition: The effects of subconscious priming on ethical behavior. Academy of Management Journal, 57, 723742.

CrossRef (https://doi.org/10.5465/amj.2011.1009)

Google Scholar (http://scholar.google.com/scholar_lookup?

title=Conscience\%20without\%20cognition\%3A\%20The\%20effects\%20of\%20subconscio us\%2opriming\%20on\%2oethical\%2obehavior\&author=DT.\%2oWelsh\&author=LD.\%20 Ord\%C3\%B3\%C3\%B1ez\&journal=Academy\%20of\%2oManagement\%20Journal\&volume $=57 \&$ pages $=723-742 \&$ publication_year $=2014$ )

Wernimont, P. F., \& Fitzpatrick, S. (1972). The meaning of money. Journal of Applied Psychology, 56, 218-226.

CrossRef (https://doi.org/10.1037/hoo33107)

Google Scholar (http://scholar.google.com/scholar_lookup? title=The\%20meaning\%20of\%20money\&author=PF.\%20Wernimont\&author=S.\%20Fit zpatrick\&journal=Journal\%20of\%20Applied\%20Psychology\&volume $=56 \&$ pages $=218-$ 226\&publication_year=1972)

Wilson, D. S., Near, D., \& Miller, R. R. (1996). Machiavellianism: A synthesis of evolutionary and psychological literatures. Psychological Bulletin, 119, 285-299. CrossRef (https://doi.org/10.1037/0033-2909.119.2.285) Google Scholar (http://scholar.google.com/scholar_lookup? title=Machiavellianism\%3A\%20A\%20synthesis\%20of\%20evolutionary\%20and\%20psyc hological\%2oliteratures\&author=DS.\%20Wilson\&author=D.\%20Near\&author=RR.\%20 Miller\&journal=Psychological\%2oBulletin\&volume $=119 \&$ pages $=285$ 299\&publication_year=1996)

Wimbush, J., Shepard, J., \& Markham, S. (1997). An empirical examination of the relationship between ethical climate and ethical behaviour from multiple levels of analysis. Journal of Business Ethics, 16, 1705-1716.

CrossRef (https://doi.org/10.1023/A\%3A1017952221572)

Google Scholar (http://scholar.google.com/scholar_lookup?

title=An\%20empirical\%20examination\%20of\%20the\%2orelationship\%2obetween\%20e thical\%2oclimate\%20and\%20ethical\%2obehaviour\%2ofrom\%2omultiple\%2olevels\%20 of\%20analysis\&author=J.\%2oWimbush\&author=J.\%20Shepard\&author=S.\%20Markha m\&journal=Journal\%20of\%20Business\%20Ethics\&volume=16\&pages=17051716\&publication_year=1997)

Wong, H. M. (2008). Religiousness, love of money, and ethical attitudes of Malaysian evangelical Christians in business. Journal of Business Ethics, 81, 169-191.

CrossRef (https://doi.org/10.1007/s10551-007-9487-0)

Google Scholar (http://scholar.google.com/scholar_lookup? title=Religiousness\%2C\%2olove\%20of\%20money\%2C\%20and\%20ethical\%20attitudes \%20of\%20Malaysian\%20evangelical\%20Christians\%20in\%2obusiness\&author=HM.\%2 oWong\&journal=Journal\%20of\%2oBusiness\%2oEthics\&volume=81\&pages=169191\&publication_year=2008)

Yamauchi, K. T., \& Templer, D. J. (1982). The development of a money attitude scale. Journal of Personality Assessment, 46, 522-528.

CrossRef (https://doi.org/10.1207/s15327752jpa4605_14) Google Scholar (http://scholar.google.com/scholar_lookup? title=The\%20development\%20of\%20a\%20money\%20attitude\%20scale\&author=KT.\%2 
oYamauchi\&author=DJ.\%20Templer\&journal=Journal\%20of\%20Personality\%20Assess ment\&volume $=46 \&$ pages $=522-528 \&$ publication_year $=1982)$

Zhang, L. Q. (2009). An exchange theory of money and self-esteem in decision making. Review of General Psychology, 13, 66-76.

CrossRef (https://doi.org/10.1037/a0014225)

Google Scholar (http://scholar.google.com/scholar_lookup? title=An\%20exchange\%20theory\%20of\%2omoney\%20and\%2oselfesteem\%20in\%2odecision\%20making\&author=LQ.\%20Zhang\&journal=Review\%20of\% 20General\%20Psychology\&volume $=13 \&$ pages $=66-76 \&$ publication_year $=2009$ )

Zhou, X. Y., \& Gao, D. G. (2008). Social support and money as pain management mechanisms. Psychological Inquiry, 19, 127-144.

CrossRef (https://doi.org/10.1080/10478400802587679)

Google Scholar (http://scholar.google.com/scholar_lookup?

title=Social\%20support\%20and\%20money\%20as\%20pain\%20management\%20mechan isms\&author $=\mathrm{XY}$ \%20Zhou\&author=DG.\%20Gao\&journal=Psychological\%20Inquiry\&v olume=19\&pages=127-144\&publication_year $=2008$ )

Zhou, X. Y., Vohs, K. D., \& Baumeister, R. F. (2009). The symbolic power of money: Reminders of money alter social distress and physical pain. Psychological Science, 20 , 700-706.

CrossRef (https://doi.org/10.1111/j.1467-9280.2009.02353.x)

Google Scholar (http://scholar.google.com/scholar_lookup?

title=The\%20symbolic\%2opower\%20of\%2omoney\%3A\%2oReminders\%20of\%2omoney \%20alter\%20social\%2odistress\%20and\%2ophysical\%2opain\&author=XY.\%20Zhou\&au thor=KD.\%20Vohs\&author=RF.\%20Baumeister\&journal=Psychological\%20Science\&vol ume=20\&pages $=700-706 \&$ publication_year $=2009$ )

\section{Copyright information}

(C) Springer Science+Business Media Dordrecht 2016

\section{About this article}

Cite this article as:

Tang, T.LP., Sutarso, T., Ansari, M.A. et al. J Bus Ethics (2018) 148: 919. https://doi.org/10.1007/s10551-0152942-4

- DOI (Digital Object Identifier) https://doi.org/10.1007/s10551-015-2942-4

- Publisher Name Springer Netherlands

- Print ISSN 0167-4544

- Online ISSN 1573-0697

- $\underline{\text { About this journal }}$

- Reprints and Permissions

\section{Personalised recommendations}


1. Enron-Era Disclosure of Off-Balance-Sheet Entities

Chandra, Uday... Stone, Mary S.

Accounting Horizons (2006)

2. Convergence of corporate governance practices in the post-Enron period:

behavioral transformation or box-checking exercise?

Bozec, Richard... Dia, Mohamed

Corporate Governance: The international journal of business in society (2012)

Want recommendations via email? Sign up now

Powered by: Recommended ${ }^{\mathbf{R}}$

\section{SPRINGER NATURE}

(C) 2017 Springer International Publishing AG. Part of Springer Nature.

Not logged in International Islamic University Malaysia (IIUM) (2000621865) - 4972 SpringerLink Malaysia eBook Consortium-2009-2010 copyright (3000134874) - 6816 SpringerLink Malaysia eJournal Consortium - Higher Education (3000155375) - 8354 Springerlink Malaysia consortium (3000519906) - 10122 SpringerLink Malaysia eJourna Consortium - Higher Education (3000716851) - SpringerLink Malaysia eJournal Consortium - Higher Education (3000916360) 210.48.222.9 